\title{
Tidal Conversion and Dissipation at Steep Topography in a Channel Poleward of the Critical Latitude
}

\author{
KenNeTH G. Hughes ${ }^{\mathrm{a}}$ \\ School of Earth and Ocean Sciences, University of Victoria, Victoria, British Columbia, Canada \\ JODY M. KLYMAK \\ School of Earth and Ocean Sciences, and Department of Physics and Astronomy, University of Victoria, \\ Victoria, British Columbia, Canada
}

(Manuscript received 20 June 2018, in final form 6 March 2019)

\begin{abstract}
In high-latitude fjords and channels in the Canadian Arctic Archipelago, walls support radiating internal tides as Kelvin waves. Such waves allow for significant barotropic to baroclinic tidal energy conversion, which is otherwise small or negligible when poleward of the critical latitude. This fundamentally three-dimensional system of a subinertial channel is investigated with a suite of numerical simulations in rectangular channels of varying width featuring idealized, isolated ridges. Even in channels as wide as 5 times the internal Rossby radius, tidal conversion can remain as high as predicted by an equivalent two-dimensional, nonrotating system. Curves of tidal conversion as a function of channel width, however, do not vary monotonically. Instead, they display peaks and nulls owing to interference between the Kelvin waves along the wall and similar waves that propagate along the ridge flanks, the wavelengths of which can be estimated from linear theory to guide prediction. Because the wavelengths are comparable to width scales of Arctic channels and fjords, the interference will play a first-order role in tidal energy budgets and may consequently influence the stability of glaciers, the ventilation of deep layers, the locations of sediment deposition, and the fate of freshwater exiting the Arctic Ocean.
\end{abstract}

\section{Introduction}

The Arctic is home to numerous channels and fjords that are typically 2-3 times wider than the internal Rossby radius (Cottier et al. 2010). Similarly, the larger channels in the Canadian Arctic Archipelago can be an order of magnitude wider than this radius (McLaughlin et al. 2004). Consequently, rotation governs hydrography and energetics. In these settings, and the ocean as a whole, internal tides generated by stratified tidal flow over obstacles are the starting point in an energy cascade from the barotropic tides to irreversible mixing (Munk and Wunsch 1998; Klymak and Gregg 2004; Inall et al. 2005; Arneborg et al. 2017). In better-studied, midlatitude fjords, energy extracted from the barotropic tide

\footnotetext{
${ }^{\text {a }}$ Current affiliation: College of Earth, Ocean, and Atmospheric Sciences, Oregon State University, Corvallis, Oregon.
}

Corresponding author: Kenneth G.Hughes, khughes@ceoas. oregonstate.edu is often the primary source for driving the mean circulation (Freeland and Farmer 1980; Inall and Rippeth 2002). Furthermore, during periods of stagnation, mixing ultimately induced by radiated tides is the only effective mechanism to mix deep water within a fjord (Stigebrandt and Aure 1989, and references therein).

The gross behavior of stratified tidal flow over an obstacle is concisely captured by three parameters (e.g., Sarkar and Scotti 2017): the conversion of barotropic to baroclinic energy, the ratio of barotropic tidal energy dissipated locally to the total lost; and the energy transported by internal waves elsewhere. These influence the location, strength, and mechanisms of mixing. Several parameters can help predict the fate of barotropic tidal energy: (i) the barotropic current speed $U$ relative to the internal wave speed over the topography; (ii) $U$ relative to the product of the obstacle height and stratification, termed the topographic Froude number (Fr); and (iii) the ratio of internal wave slope to topographic slope $\gamma$. The last parameter is ill defined in locations poleward of the critical latitude where 

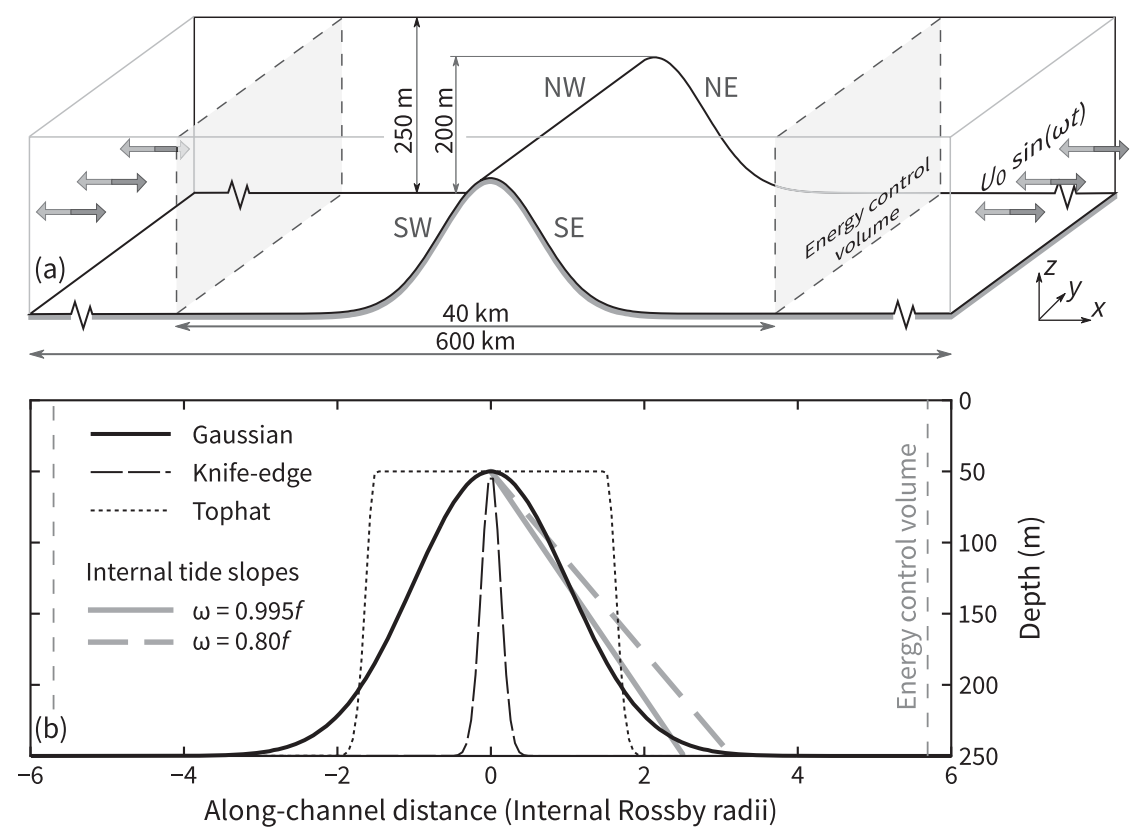

FIG. 1. Domain, topographies, and notation used in this study. Note that, as evident from the wave slopes, the ends of the energy control volume are approximately one mode- 1 wavelength from the center.

the Coriolis frequency $f$ exceeds the tidal frequency $\omega$. This leads to a fourth important parameter: whether the tide is super- or subinertial.

In the two-dimensional, linear limit, freely propagating internal tides exist only equatorward of the critical latitude $\left(30.0^{\circ}\right.$ for $\mathrm{K}_{1}$ and $74.5^{\circ}$ for $\left.\mathrm{M}_{2}\right)$. These are termed superinertial internal tides. Linear tidal conversion drops quickly as this latitude is approached, decreasing as $\sqrt{1-(f / \omega)^{2}}$ (Llewellyn Smith and Young 2003). Poleward of the critical latitude, still in two dimensions, there is an evanescent response. Tidally averaged conversion, however, only results from nonlinearities and turbulence because linear perturbations in pressure and cross-ridge velocity are $90^{\circ}$ out of phase (see, e.g., Musgrave et al. 2016). Consequently, the fraction of energy lost from the barotropic tide that is dissipated locally is close to 1 , but the amount of energy extracted from the barotropic tide in absolute terms is small compared to low and midlatitudes.

Two types of propagating waves exist that allow for a nonzero tidally averaged conversion: (i) coastal trapped waves (e.g., Huthnance et al. 1986), a classification that includes internal Kelvin waves as a limiting case, and (ii) high-frequency internal waves generated by nonlinearities. Rippeth et al. (2017) observe and simulate packets of high-frequency internal waves generated by internally supercritical flow at the edge of Spitsbergen Bank $\left(75^{\circ} 30^{\prime} \mathrm{N}\right)$ and suggest this is a key mechanism linking tides to turbulence poleward of the critical latitude. Støylen and Fer (2014) describe similar highfrequency wave packets in Van Mijenfjorden, Svalbard $\left(77^{\circ} 45^{\prime} \mathrm{N}\right)$, but in this case, the fjord setting also permits internal Kelvin waves at the tidal frequency and these were found to transport the majority of the internal wave energy away from the sill.

Internal Kelvin waves behave as nonrotating internal waves in the direction in which they travel. Therefore, it is reasonable to expect that much of the literature for the two-dimensional ( $x-z$, across-topography-vertical) case is applicable to this paper in which we consider an isolated bump that is independent of $y$ (Fig. 1). Specifically, we expect tidal conversion to scale as $U_{0}^{2}$ and $N$ where $U_{0}$ and $N$ are the tidal current amplitude far from the obstacle and the buoyancy frequency near the crest of the obstacle, respectively. Further, the majority of the energy flux is likely contained in the first internal mode (Llewellyn Smith and Young 2003; St. Laurent et al. 2003). These scalings, however, provide no clues as to how conversion will scale with channel width in a subinertial channel in which the dominant response, a Kelvin wave, has a cross-channel structure.

The simulated conversion in a channel is influenced strongly by topographically trapped internal wave interference as will be shown later. Curiously, interference also occurs in many nominally two-dimensional scenarios. For example, conversion is controlled by the distance between peaks in idealized, periodic topography 
TABLE 1. Summary of all simulations undertaken. Bolded values identify the combination of parameters used in the example in section 3 .

\begin{tabular}{|c|c|c|c|}
\hline Topography & Channel widths $(\mathrm{km})$ & $U_{0}\left(\mathrm{~cm} \mathrm{~s}^{-1}\right)$ & $\omega / f$ \\
\hline \multicolumn{4}{|c|}{ Variable channel width (sections 3,5 , and $6 a$ ) } \\
\hline Gaussian & $\begin{array}{c}0.2,0.4,0.6,0.8,1.2,1.6,2,3,4,6,7,8,10 \\
12,13,14,15,16,19,23, \mathbf{2 6}, 29,32,37,42 \\
48,56,65\end{array}$ & 4 & $0.80, \mathbf{0 . 9 9 5}$ \\
\hline Tophat & & 4 & $0.80,0.995$ \\
\hline Knife-edge & & 4 & $0.80,0.995$ \\
\hline \multicolumn{4}{|c|}{ Variable frequency (section $4 c$ ) } \\
\hline Gaussian & 40 & 4 & $\begin{array}{c}0.70,0.75,0.80,0.85,0.90 \\
0.95,0.995,1.05\end{array}$ \\
\hline \multicolumn{4}{|c|}{ Variable forcing (section 6b) } \\
\hline Gaussian & 26 & $\begin{array}{c}1,2,4,6,9,12 \\
16,20,24\end{array}$ & 0.995 \\
\hline
\end{tabular}

(Balmforth and Peacock 2009). A realistic example of this occurs in Luzon Strait where strong conversion results from constructive interference of semidiurnal internal tides generated at approximately parallel ridges (Buijsman et al. 2012; Klymak et al. 2013). A related example, albeit only significant for short (small fractional height) obstacles, is conversion by a tophat ridge, where the distance between the ends of the tophat is a controlling factor (St. Laurent et al. 2003). An example of interference that involves subinertial diurnal internal tides occurs at Izu-Oshima Island near Japan $\left(34^{\circ} \mathrm{N}\right)$ where the natural period for an internal Kelvin wave circumnavigating the $52-\mathrm{km}$-circumference island is close to the diurnal tidal period. Masunaga et al. (2017) demonstrate that the forcing tidal frequency controls the Kelvin wave magnitude. Mihanović et al. (2014) provide similar results for a $5-\mathrm{km}$-circumference island in the Adriatic Sea $\left(42^{\circ} \mathrm{N}\right)$.

In this paper, we undertake a suite of three-dimensional numerical simulations of tidal flows in a rectangular channel over three idealized topographies (section 2). The initial, qualitative results (section 3) demonstrate the controlling role of the internal waves that travel along the ridge flanks. Details of these waves are far from trivial (section 4) and cause complicated but predictable changes in tidal conversion as channel width varies (section 5). The importance of tidal conversion to the barotropic energy budget and the subsequent fate of baroclinic energy is evaluated (section 6) and leads to discussions on the practical relevance of this idealized study and the importance of three-dimensionality for subinertial tides (section 7).

\section{Methods}

\section{a. Geometry and forcing}

Several three-dimensional simulations (Table 1) of stratified tidal flow over an idealized obstacle are undertaken with the MITgem (Marshall et al. 1997; Adcroft et al. 2004) together with an online barotropicbaroclinic energy decomposition (Kang and Fringer 2012; Klymak et al. 2016). The north-south aligned obstacle lies at the center of a $600-\mathrm{km}$-long east-west channel (Fig. 1a). Our analysis will concentrate on a Gaussian obstacle, with knife-edge and tophat simulations providing further clarification (Fig. 1b). Such obstacles are frequently used in two-dimensional studies (e.g., St. Laurent et al. 2003; Llewellyn Smith and Young 2003; Klymak et al. 2010, 2013; Musgrave et al. 2016).

The chosen dimensions and oceanographic parameters are loosely based on a particular sill in the Canadian Arctic Archipelago where internal Kelvin waves have been observed (Hughes et al. 2018). Specifically, a 200-m-tall obstacle sits in $250 \mathrm{~m}$ of water with a constant stratification of $N=6.2 \times 10^{-3} \mathrm{~s}^{-1}\left(\Delta \rho=1.0 \mathrm{~kg} \mathrm{~m}^{-3}\right.$ over $250 \mathrm{~m}$ ). The obstacle is always steeper than a nonrotating internal tide slope (Fig. 1b).

Within $20 \mathrm{~km}$ either side of the obstacle crest, the model grid spacing is fixed at $d x=200 \mathrm{~m}$. Beyond this, grid spacing increases $1.5 \%$ per cell. In the cross-channel direction, $d y$ is constant at $200 \mathrm{~m}$ and small relative to the internal Rossby radius $L_{R}=N H / \pi f=3500 \mathrm{~m}$. Vertical grid spacing is also constant $(60$ cells, $d z=$ $4.2 \mathrm{~m}$ ). Given the 48:1 aspect ratio of grid cells, all simulations are hydrostatic (see Berntsen et al. 2009).

Tidal currents are forced at the boundaries using $100-\mathrm{km}$-long velocity sponges with relaxing time scales of 100 and $1000 \mathrm{~s}$ at the outer and inner edges of the sponges, respectively. To produce a progressive tidal wave, we include a phase difference between the two sponges of $600 \mathrm{~km} / \sqrt{g \bar{H}} \approx 12000 \mathrm{~s}$, where $\bar{H}$ is the mean depth. (Simulations were initially undertaken without the phase difference and produced equivalent results, but we do not include these.) Furthermore, tidal forcing is linearly ramped over the first two tidal cycles. 
In most simulations, tidal currents in the deep water are kept moderate $\left(4 \mathrm{~cm} \mathrm{~s}^{-1}\right)$ to produce predominantly, but not perfectly, linear behavior. Larger-amplitude tides are considered in section $6 \mathrm{~b}$. Counteracting the tidal forcing is the induced form drag and quadratic bottom drag $\left(C_{d}=\right.$ $\left.1 \times 10^{-3}\right)$. A free slip condition with no drag is applied at the walls. Viscosity and diffusivity are parameterized based on density overturns (Klymak and Legg 2010) with background values of $1 \times 10^{-5} \mathrm{~m}^{2} \mathrm{~s}^{-1}$ for both parameters.

All simulations use a single tidal constituent, with most simulations using a period of either $44640 \mathrm{~s}$ or $55800 \mathrm{~s}$. The former is a close approximation to the $\mathrm{M}_{2}$ tidal period and the latter is simply a hypothetical tide with a period $25 \%$ longer. The Coriolis frequency is set based on a latitude of $76^{\circ}$, slightly poleward of the critical latitude for our approximate $\mathrm{M}_{2}$ tide $\left(74.8^{\circ}\right.$, $\omega=0.995 f)$ and well poleward for the longer period tide $\left(50.5^{\circ}, \omega=0.80 f\right)$. Both tidal periods conveniently divide into several integer time steps. Here $d t=10 \mathrm{~s}$ and easily satisfies the CFL criterion for internal waves, which travel at $\leq 0.5 \mathrm{~m} \mathrm{~s}^{-1}$. Conversely, surface waves travel at $50 \mathrm{~m} \mathrm{~s}^{-1}$, so an implicit free surface is used.

\section{b. Barotropic-baroclinic energy decomposition}

We treat the fate of barotropic (the depth-mean component) and baroclinic (the remaining component) tidal energy in a tidally steady system following Kang and Fringer (2012) with some simplifications to be described. Barotropic tidal energy is lost to either bottom boundary layer dissipation or converted to baroclinic energy. After conversion, energy may (i) leave the system as an internal wave flux, (ii) dissipate in the interior, or (iii) dissipate in the bottom boundary layer. The vertically integrated energy budgets $\left(\mathrm{W} \mathrm{m}^{-2}\right)$ become

$$
\begin{aligned}
& \nabla_{H} \cdot\left\langle\overline{\mathbf{F}_{0}}\right\rangle=-\langle\bar{C}\rangle-\left\langle D_{0}\right\rangle, \\
& \nabla_{H} \cdot\left\langle\overline{\mathbf{F}^{\prime}}\right\rangle=\langle\bar{C}\rangle-\left\langle D^{\prime}\right\rangle-\langle\bar{\varepsilon}\rangle,
\end{aligned}
$$

where $\mathbf{F}$ is a tidal energy flux, $C$ is conversion from barotropic to baroclinic energy, $D$ is bottom boundary layer dissipation, and $\varepsilon$ is turbulent dissipation in the interior. A subscript 0 and a prime denote barotropic and baroclinic components, respectively, $\nabla_{H}$ is horizontal divergence, overbars denote depth integrals, and angle brackets denote tidal averages. With the exception of section 3 where snapshots are shown, all tidal energy terms we present are averaged over the seventh tidal cycle.

For most of the paper, we decompose baroclinic and barotropic motions by removing the depth-mean velocity for ease of online calculation. This decomposition, however, is not formally valid over a sloping seafloor, and instead spatial modes must be solved for in the vertical and horizontal (Brink 2006, section 4). Qualitatively, the informal decomposition is expected to be similar to the formal decomposition, but will usually underestimate the energy flux in "internal" motions because the formal modes have a depth-mean component (Musgrave 2018, manuscript submitted to J. Phys. Oceanogr.). Where we quantify energy budgets below we are careful to only integrate internal wave energy fluxes over portions of the domain where the seafloor is flat.

The conversion term $C$ is a focus of this paper and the link between the individual energy budgets for barotropic and baroclinic energies. It is dominated by its linear, hydrostatic component:

$$
C_{\text {lin }}=\overline{\rho^{\prime} g W},
$$

where $\rho^{\prime}$ is a density perturbation, and $W=W(z)$ is the vertical velocity that results from convergence or divergence of horizontal barotropic flow between the seafloor and $z$. Our volume-integrated budgets additionally include the small unclosed components of $C$ documented by Kang [2011, see Eq. (5.89)].

After conversion, baroclinic energy either dissipates locally (defined as within the 40-km-long energy control volume centered about the obstacle as in Fig. 1a) or propagates out of the system. The component that leaves is described concisely by the depth-integrated baroclinic flux $\overline{\mathbf{F}^{\prime}}$ :

$$
\overline{\mathbf{F}^{\prime}}=\overline{\mathbf{u}^{\prime} p^{\prime}}
$$

where $p^{\prime}$ is the pressure perturbation relative to a horizontally independent background state and $\mathbf{u}^{\prime}$ is the velocity with the depth average removed. We have ignored the advective and diffusive components of $\overline{\mathbf{F}^{\prime}}$ as these are negligible here.

Bottom boundary layer dissipation is decomposed into barotropic and baroclinic components:

$$
\begin{aligned}
& D_{0}=\rho_{0} C_{d}\left|\mathbf{u}_{H}\right|(u U+v V), \\
& D^{\prime}=\rho_{0} C_{d}\left|\mathbf{u}_{H}\right|\left(u u^{\prime}+v v^{\prime}+w^{2}\right) .
\end{aligned}
$$

Uppercase, lowercase, and primed velocities are the barotropic, complete, and baroclinic components, respectively. A subscript $H$ denotes the horizontal component.

Turbulent dissipation $\varepsilon$ is not decomposed into barotropic and baroclinic components. Doing so is arguably beyond the point of diminishing returns. Instead, we have placed $\varepsilon$ in the baroclinic energy budget as we 


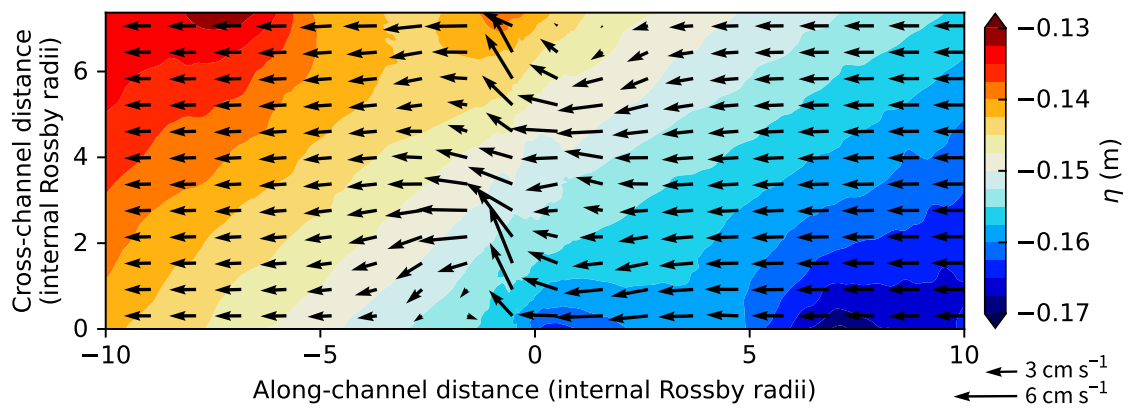

FIG. 2. A snapshot of barotropic velocities and sea surface height after seven tidal cycles.

expect that it predominantly results from processes occurring after conversion to baroclinic energy.

\section{Flow behavior}

Much of the behavior of the stratified tidal flow is apparent in snapshots of barotropic fields (Fig. 2), baroclinic fields (Fig. 3) and energy components (Fig. 4). In this section, we describe this behavior for a moderately wide $\left(26 \mathrm{~km}, 7.3 L_{R}\right)$ channel. This channel is geometrically comparable to Van Mijenfjorden in Svalbard (10-14 km wide), which has been studied observationally (Støylen and Fer 2014) and thereby provides some qualitative validation of the processes we study here.

Outside the vicinity of the obstacle, the barotropic current is spatially uniform because the channel is much narrower than the external Rossby radius $(350 \mathrm{~km})$. Although it is not obvious in Fig. 2, to first order the sea surface height throughout the length of the channel behaves as a plane, progressive wave traveling west to east. Superimposed on this plane wave is an anomaly that proceeds counterclockwise around the center of the channel, akin to an amphidromic point. The relationship between sea surface height and convergence and divergence, however, is often counterintuitive, both because a whole wave cycle must be considered and because it depends on baroclinic processes to be described. Some intuition, nevertheless, can be gained from the similar problem of the development of the steady state flow of unstratified fluid over an obstacle in a wide channel, which is analyzed in detail in chapter 3 of Pratt and Whitehead (2008).
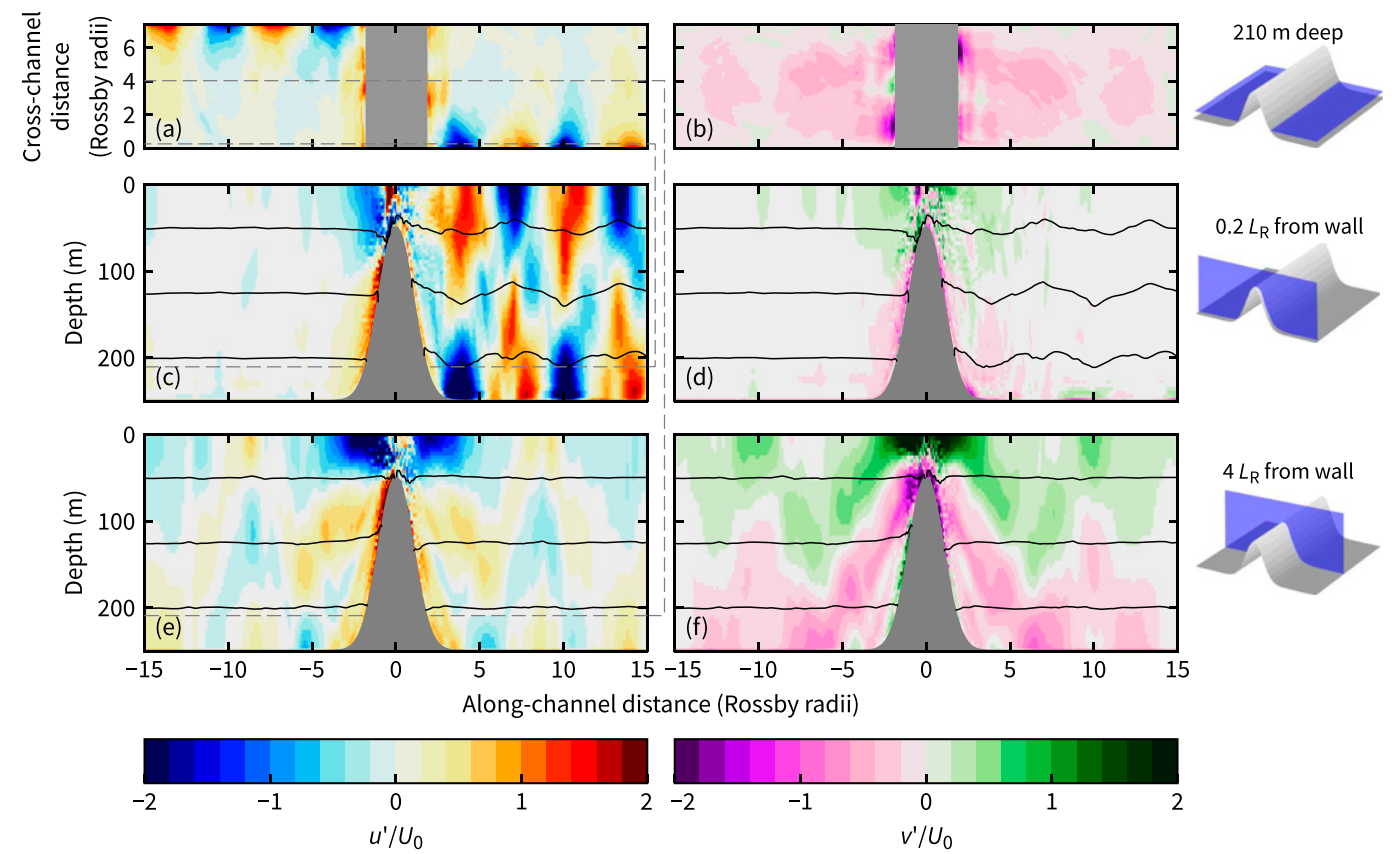

FIG. 3. A snapshot of baroclinic velocities and isopycnals after seven tidal cycles. Tidal forcing in the deep water has an amplitude of $U_{0}=4 \mathrm{~cm} \mathrm{~s}^{-1}$ and a frequency of $\omega=0.995 f$. 


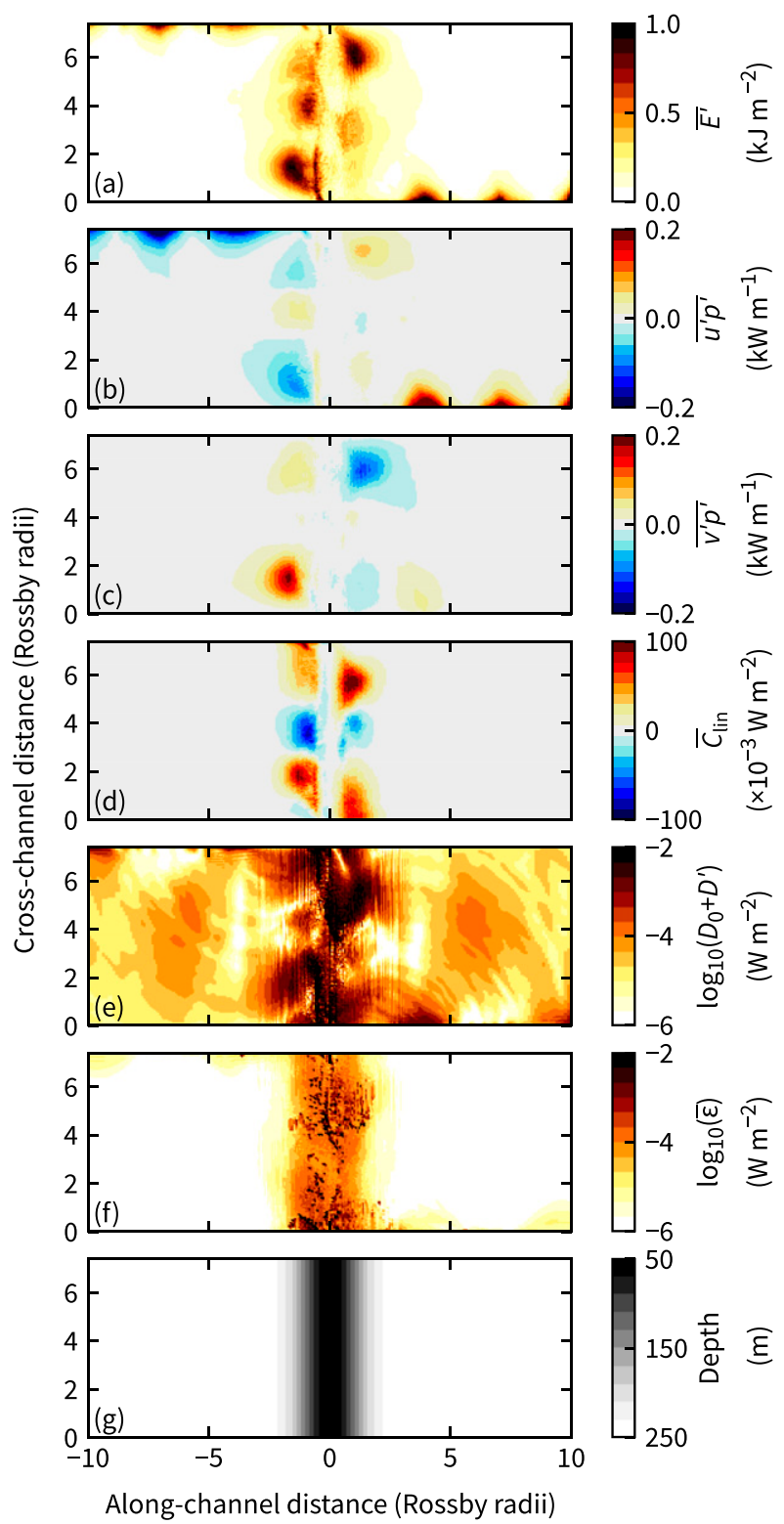

FIG. 4. Snapshots of energy terms after seven tidal cycles. Corresponding fields are shown in Fig. 3. (a) Baroclinic energy density. (b),(c) Eastward and northward energy flux via internal waves. (d) The linear component of the conversion rate. (e) Bottom boundary layer dissipation. (f) Turbulent dissipation due to overturning. (g) Obstacle bathymetry.

Internal Kelvin waves are the dominant signal in the along-channel baroclinic velocity fields near the boundary. The $e$-folding scale of 1 Rossby radius is evident in map view (Fig. 3a) and their equivalence to nonrotating internal waves is evident in cross section (Fig. 3c); that is, they are beam-like with slopes of $\sqrt{\left(N^{2}-\omega^{2}\right) / \omega^{2}}$, which equates to approximately 0.023 for the $\omega=0.995 f$ tide or equivalently $3.1 L_{R} / 250-\mathrm{m}$ depth. Note that the beams in Fig. 3 are diffuse because low-mode waves dominate; high-mode waves propagate away from the topography more slowly and dissipate locally (Klymak et al. 2010).

The response away from the wall is a combination of the expected linear, subinertial response and transient near-inertial waves (Figs. 3e,f), with the latter resulting from the initialization of the model from rest. Linear theory for the two-dimensional subinertial case predicts that baroclinic velocities decay exponentially from the obstacle with an inverse decay scale $\hat{k}$ (Musgrave et al. 2016):

$$
\hat{k}=\frac{\pi}{H N} \sqrt{f^{2}-\omega^{2}} .
$$

For our chosen parameters, $\hat{k}$ corresponds to a decay scale of $34 \mathrm{~km} \approx 10 L_{R}$. This scale is somewhat apparent in Figs. $3 \mathrm{e}$ and $3 \mathrm{f}$ as the general decay in baroclinic velocities along the center of the channel. However, it is obscured by the transient, near-inertial waves.

Looking at these fields from an energy perspective (Fig. 4) effectively filters out the near-inertial waves as these have negligible associated horizontal energy fluxes. Baroclinic energy density and fluxes (Figs. 4a-c) dominate in two areas: on the flanks of the topography and beside the walls on the right-hand side. Details of the waves traveling along the flanks are discussed in section 4 . Here we simply note that they are generated by convergence and divergence at the northeast and southwest parts of the bump and because they are traveling beside an inclined boundary, the baroclinic energy density decays more slowly in the $x$ direction (compared to away from the vertical walls in the $y$ direction). Because this wave includes a depth-mean component (see sections $2 \mathrm{~b}$ and 4 ), the subinertial wave fluxes along the ridge in Fig. $4 \mathrm{c}$ are underestimated by approximately $25 \%$. This underestimate, derived from the mode shapes described in section 4 , is a function of the obstacle shape and tidal frequency. Among all obstacles and tidal frequencies considered in this study, underestimates of the subinertial wave fluxes along the ridge are approximately $25 \%-50 \%$.

The internal waves (at the tidal frequency) along the flanks explain much of the conversion term's spatial distribution (Fig. 4d). For the case shown, the width of the channel is 1.8 times the wavelength of the alongflank wave. When this wave interacts with the vertical velocity induced by the barotropic tidal forcing, it leads to lobes of positive and negative conversion. During this snapshot, there is one negative and two positive lobes on each side of the obstacle. The same is true for maps of tidally averaged conversion (not shown). Negative conversion is typically explained as resulting 
from interaction between remotely and locally generated internal tides (e.g., Kang and Fringer 2012; Fer et al. 2015; Masunaga et al. 2017). In our case, however, it is better described as the interaction between the currently generated and previously generated internal tides.

Bottom boundary layer dissipation $\left(D_{0}+D^{\prime} ;\right.$ Fig. $\left.4 \mathrm{e}\right)$ is the only term shown that is not concentrated either near the wall or near topography. For example, wave interference, including with the barotropic tide, leads to locally large and small velocities and is responsible for much of the observed distribution. A radial pattern is also evident and occurs because waves are generated as the along-obstacle waves scatter at the northwest and southeast ends of the obstacle. These effects are of minor importance, however, as dissipation levels away from the walls or the obstacle are comparable to what they would be in an equivalently forced system without topography.

Most turbulent dissipation in the interior water column occurs over the obstacle where high-mode waves dissipate and strong nonlinearities occur. Appreciable levels of turbulence also occur along walls. Vertical slices near the walls (not shown) show that turbulence is strongest at middepth where isopycnal perturbations are largest and near the seafloor where baroclinic convergence induces instabilities.

\section{Structure of the along-obstacle waves}

The along-obstacle waves are responsible for the spatial pattern of tidal conversion as described in the previous section. Moreover, the extent to which the positive and negative regions cancel controls how conversion and divergences of barotropic and baroclinic flux vary: curves of these quantities as a function of channel width display peaks and nulls (Fig. 5) rather than varying monotonically as may be reasonably expected. In this section, we develop a linear model of the along-obstacle waves, which are conceptually similar to internal Kelvin waves beside a wall and a step. As background, we first recap the properties of such waves.

\section{a. Internal Kelvin waves}

If an internal Kelvin wave is traveling parallel to a vertical wall at $x=0$ and is hydrostatic and geostrophically balanced, then velocities normal to the wall are identically zero and the wave solution is separable in $y$ and $z$. For example, the mode- 1 alongchannel velocity is

$$
v^{\prime}=\mathscr{T}(z) \exp \left[i\left(\omega t-k_{y} y\right)\right] \exp \left(-x / L_{R}\right),
$$
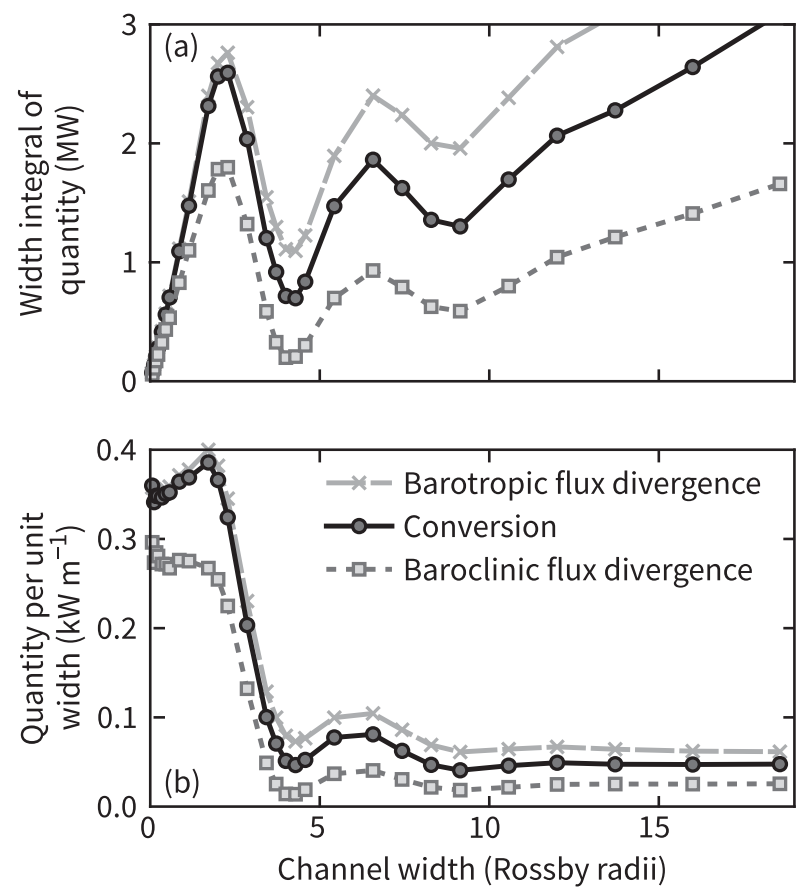

FIG. 5. Tidal conversion and associated quantities in channels of varying width with a Gaussian obstacle. The resulting baroclinic flux divergence from the energy control volume is smaller owing to local dissipation.

where $\mathscr{V}(z)$ is a mode shape. Tidal frequency $\omega$ and along-channel wavenumber $k_{y}$ are related by the hydrostatic dispersion relation:

$$
\omega=\frac{k_{y} N}{k_{z}} .
$$

With constant buoyancy frequency, the mode- 1 vertical wavenumber would be $k_{z}=\pi / H$ and the mode- 1 component of $\mathscr{V}$ would be $\cos (\pi z / H)$ multiplied by some coefficient. The along-channel wavelength then follows as

$$
\lambda_{y}=\frac{2 \pi}{k_{y}}=\frac{2 N H}{\omega} .
$$

These waves are nondispersive:

$$
c_{p}=\frac{\omega}{k_{y}}=c_{g}=\frac{\partial \omega}{\partial k_{y}}=\frac{N H}{\pi} .
$$

\section{b. Step-trapped Kelvin waves}

An internal Kelvin wave can still exist if the vertical wall is replaced by a step change in depth. Following Durran (2000), we term this a step-trapped Kelvin wave. Below the step on the deeper side, the structures of the mode- 1 pressure, density, and velocity perturbations 


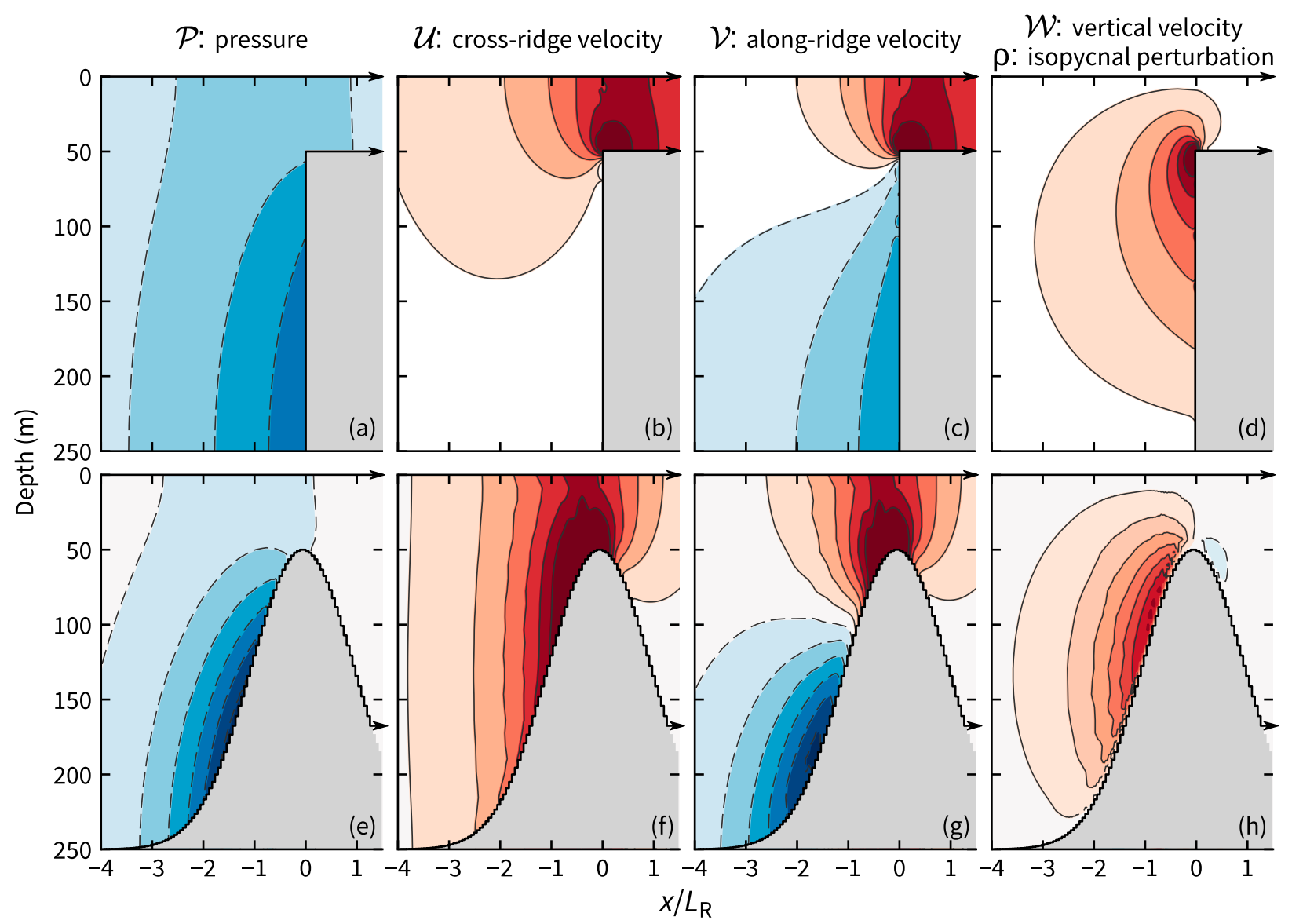

FIG. 6. Mode shapes for step- and ridge-trapped Kelvin waves traveling into the page. Hue indicates phase and saturation indicates magnitude. In both cases, a tidal frequency of $0.8 f$ is used and the domains extend indefinitely each side of the obstacle. Note that although color scales are somewhat arbitrary, $\mathscr{C}$ and $\mathscr{V}$ for a given obstacle use the same scale.

(Figs. 6a-d) resemble the bottom half of a standard internal Kelvin wave. Key differences, however, are that there is no zero crossing in $\mathscr{P}$, the zero crossing in $\mathscr{V}$ now occurs near the step height, not middepth, and the solution is no longer separable in $x$ and $z$. Wave solutions are now of the form

$$
v^{\prime} \propto \mathscr{T}(x, z) \exp \left[i\left(\omega t-k_{y} y\right)\right] .
$$

Mode shapes $\mathscr{P}, \mathscr{C}, \mathscr{T}$, and $\mathscr{W}$ must be calculated numerically as described by Chapman (1982) in detail in his Appendix B, in which he uses the term "subinertial geophysical model" rather than step-trapped Kelvin wave. The equivalent problem for an atmospheric context, which differs only by the upper boundary condition, is addressed by Samelson (1999) and Durran (2000). We briefly outline the method below.

The problem is constructed by matching both the cross-step velocity and pressure perturbation above the step at $x=0$ and requiring no normal flow at $x=0$ below the step. Taking the pressure and velocity to be the sum of a large number of Fourier modes, say $M=20$, the problem becomes a generalized eigenvalue equation of the form

$$
\mathbf{L A}=\omega^{*} \mathbf{R A},
$$

where $\mathbf{L}$ and $\mathbf{R}$ are $M \times M$ matrices derived from the matching conditions, $\mathbf{A}$ is a vector of $M$ Fourier coefficients, and the frequency $\omega^{*}$ (nondimensionalized by $f$ ) is the eigenvalue.

For our purposes, two complications arise in the matrix problem. First, $\omega^{*}$ appears when constructing $\mathbf{L}$ and $\mathbf{R}$ making it nonlinear. Second, the problem assumes $k_{y}$ is known and $\omega$ is unknown, which is the opposite of what is needed here. These two problems are overcome by solving the eigenvalue problem iteratively. First, the desired $\omega^{*}$ is set together with a broad estimate for $k_{y}$. Solving the eigenvalue problem produces an $\omega^{*}, k_{y}$ pair in which $k_{y}$ is the estimate, but $\omega^{*}$ is now the eigenvalue not the set value. The process is repeated with an 
adjusted estimate of $k_{y}$ based on linear or quadratic fits through $\omega^{*}, k_{y}$ pairs from past iterations until the answer converges to a desired accuracy. Fewer than 10 iterations will typically produce results accurate to four significant figures.

\section{c. Ridge-trapped Kelvin waves}

An internal wave trapped at an arbitrarily shaped ridge is similar in most respects to a step-trapped wave (Fig. 6), with two important, related differences. Where a slope exists, fluid parcels may travel up and down leading to a nonzero cross-ridge velocity below the crest. In weak stratification the waves are similar to topographic Rossby waves.

Solving for the mode shapes is not straightforward. Although the problem is analogous in many ways to coastal trapped waves, the literature for these waves often requires that the topography intersect the coast (e.g., Huthnance 1975, 1978; Dale and Sherwin 1996; Johnson and Rodney 2011). Conversely, codes by Brink (2006) allow for an open boundary but cannot be used here because they produce spurious solutions when $\omega \approx f$. Dale et al. (2001) overcome this issue for the coastal problem by formulating it without a velocity boundary condition that is singular. This singularity does not arise in the method used to calculate the step-trapped wave (section 4b), which we therefore generalize in this section.

A smoothly sloped ridge is approximated by an arbitrary number of flat-bottomed regions. For each region, as for the step-trapped wave, the pressure and crossridge velocity perturbations are expressed as a sum of orthogonal vertical modes and are matched at each step. Kelly et al. (2013) use such a method for superinertial tides across, rather than along, topography. Ultimately, a block matrix equation arises that is conceptually similar to Eq. (13). Again, this is solved iteratively to determine the Fourier coefficients and wavenumber. Details are provided in the appendix. ${ }^{1}$ An example of the mode shapes for the Gaussian topography are shown in Figs. 6e-h. The apparently smooth obstacle is actually comprised of 150 flatbottomed regions, each $200 \mathrm{~m}$ wide, corresponding to the resolution used in the simulations.

Knowing mode shapes elucidates the physics of the along-obstacle waves, but is ultimately a by-product of our aim to predict the wavelength for a given obstacle as this controls how conversion varies with channel width (to be described in section 5). The dispersion curves for all obstacles (Fig. 7) demonstrate that for $\omega \approx f$ the

\footnotetext{
${ }^{1}$ Our Python implementation is available at github.com/hugke729/ RidgeTrappedWave.
}

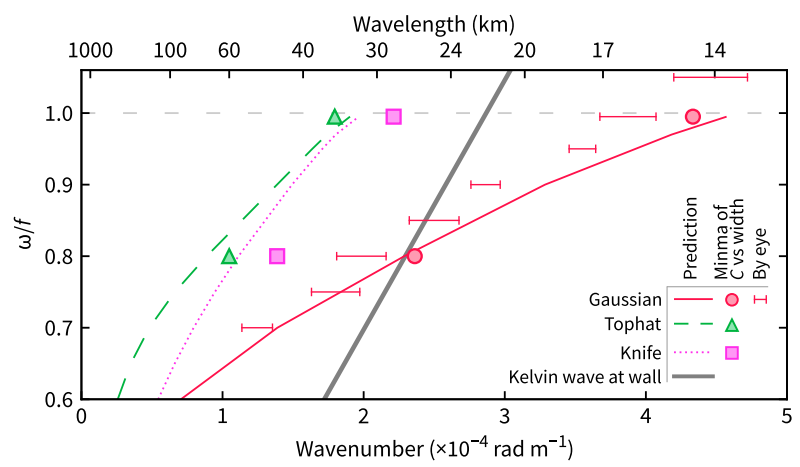

FIG. 7. Dispersion curves for the various along-obstacle waves. The curves predicted by the linear, mode-matching method are validated by the markers, which are derived from simulations.

Gaussian obstacle induces waves typically half as long as the other obstacles. Because of the sloped seafloor, the particles near the obstacle oscillate more horizontally than if they were beside a vertical wall, thereby effectively reducing the restoring force and hence the phase speed and wavelength. If frequency is decreased, say $\omega / f \lesssim 0.7$, the depth-mean component of the wave plays a larger role and consequently the width scale of the topography is a primary control on the alongobstacle wavelength.

From our suite of simulations, there are two methods by which we can validate the predicted wavelength. The simpler method is to estimate the wavelength "by eye" from model snapshots in a wide channel like those shown in Figs. 8c and 8d. We used this method for eight frequencies for the Gaussian obstacle. [The largestfrequency simulation, $1.05 f$, confirms an expectation that ridge-trapped waves can exist at slightly superinertial frequencies and that there is a continuous transition across $\omega=f$ (see Dale et al. 2001).] The by eye estimates follow the prediction well, albeit with a systematic difference that likely reflects the limitation of the estimate rather than the prediction. The second, more accurate wavelength estimate comes from the minima of a curve of conversion versus channel width. Figure 5a provides an example for the Gaussian obstacle at $\omega=0.995 f$. The wavelength in this case is $4.0 L_{R}(14 \mathrm{~km})$.

In general, the predicted wavelengths agree well with those estimated from the simulations, even for $\omega \approx f$ cases. We are not clear, however, what causes the small discrepancies. For example, the predicted wavelength for the tophat obstacle forced at $0.8 f$ is $70 \mathrm{~km}$, whereas the simulations indicate it is $60 \mathrm{~km}$.

\section{Conversion versus channel width}

The efficiency of tidal conversion, or similarly the divergence of barotropic or baroclinic flux, depends on 


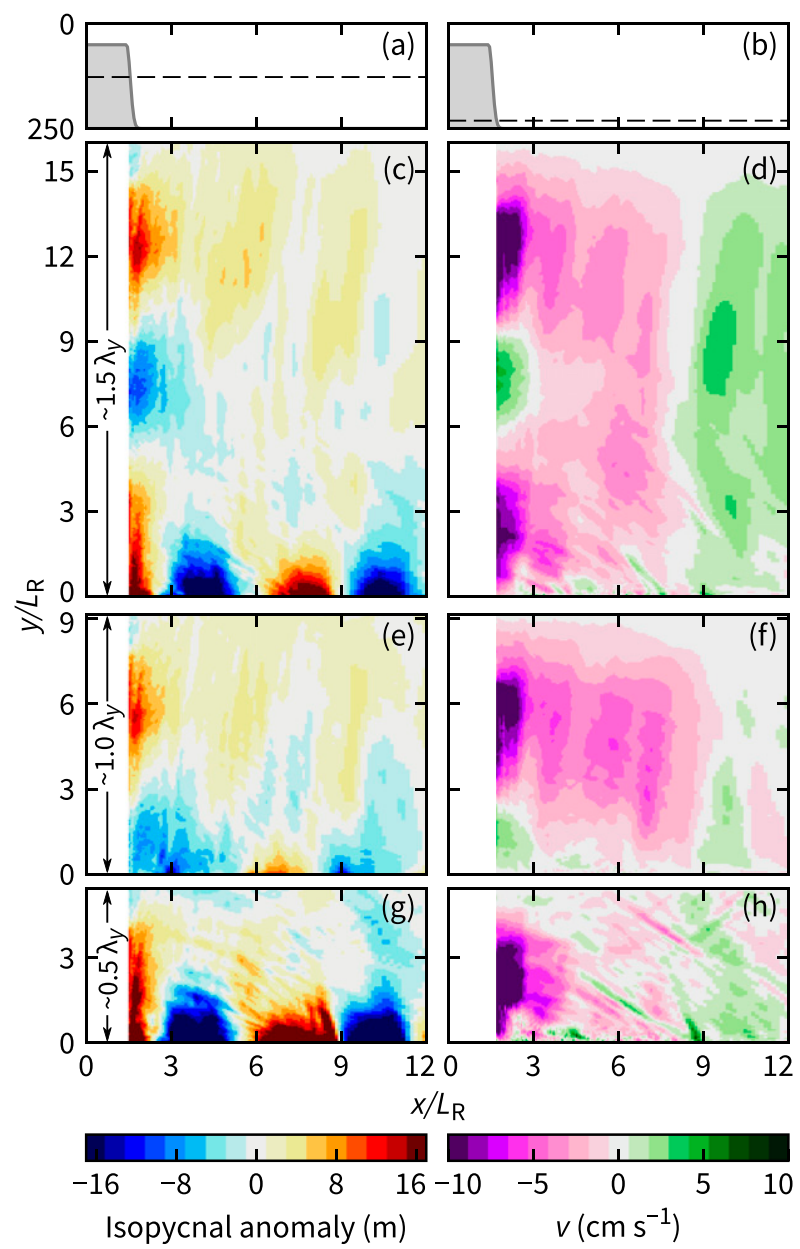

FIG. 8. Snapshots of the differing responses in three channels with large, small, and large tidally averaged conversion, respectively. Fields are taken from the eastern half of the tophat simulations after six tidal cycles at the depths shown in (a) and (b); $U_{0}=4 \mathrm{~cm} \mathrm{~s}^{-1}$ and $\omega=0.995 f$.

the channel width scaled by the along-obstacle wavelength, a result alluded to in Fig. 5. For each obstacle, curves of per unit width conversion for the two tidal frequencies approximately collapse when this width scaling is applied. This occurs despite changes of $50 \%$ or more in the along-obstacle wavelength between the two frequencies.

\section{a. Interference of the ridge-trapped and Kelvin waves}

In this section, we invoke a simple yet effective assumption from which the width dependence can be derived: internal tides are generated separately at each of the four locations denoted SE, SW, NE, and NW in Fig. 1. Given the symmetry, it suffices to consider only $\mathrm{NE}$ and SE. At both of these locations, the rotating flow in the presence of the channel walls causes periodic convergence and divergence in the $y$ direction near the obstacle and therefore an internal tide. (Narrow channels, in which the NE and SE generation regions effectively overlap, are more complicated and treated in section $5 \mathrm{~d}$.)

If, instead of a channel, only the northern wall existed and was joined by an infinitely long ridge, then on the eastern side of the obstacle, the only internal tide would be the ridge-trapped wave generated at NE traveling southward. Similarly, if only the southern wall existed, then only the Kelvin wave against the wall would occur. However, because the ridge-trapped wave travels toward SE, the Kelvin wave generation point, the two internal tides interact.

Hereafter, we use the term interference to describe the interaction at SE of (i) the propagating ridge-trapped wave with (ii) the Kelvin wave being actively generated by the barotropic tide. We assume the mode shapes of the ridge-trapped and Kelvin waves are sufficiently similar that if they meet in phase, they will constructively interfere and vice versa. Perfect destructive interference corresponds to no Kelvin wave generation: the Kelvin wave is effectively canceled as soon as it is generated.

An equivalent description of energy redistribution at SE can be given in terms of interaction between the incoming ridge-trapped wave and the forced barotropic tidal flow. The relative phases of these two waves will govern the energy transferred from the ridge-trapped wave to the barotropic tide (negative conversion) and the energy transferred to the Kelvin wave. This is analogous to two-dimensional superinertial settings in which internal tide generation at one site can be enhanced or suppressed depending on the phase of a remote, incoming internal tide (Kelly and Nash 2010; Klymak et al. 2013).

\section{b. Examples of strongly and weakly responding channels}

Consider the qualitative comparison between strongly and weakly responding channels with a tophat obstacle (Fig. 8). When the channel width is $1.5 \lambda_{y}$, isopycnal perturbations within the Kelvin wave along the southern edge reach $20 \mathrm{~m}$. Much the same picture also arises for a $0.5 \lambda_{y}$-width channel. Conversely, when the channel width is $1.0 \lambda_{y}$, the Kelvin wave is barely evident. The magnitudes of the waves traveling along the obstacle in the $1.5 \lambda_{y}$ and $1.0 \lambda_{y}$ cases are comparable as these waves have yet to interfere with those generated at SE.

Regardless of channel width, the waves at NE and SE will be generated with opposite phases. The barotropic tide is approximately a plane wave (section 3) meaning barotropic flow will diverge at one end of the obstacle and simultaneously converge at the other, and vice versa a half period later. The relative phases of the two waves when they interfere at SE depends on the 
travel time of the ridge-trapped wave. This wave travels $1 \lambda_{y}$ in one tidal period $\left(c_{p}=\lambda_{y} / T\right)$, so the waves in the $1 \lambda_{y}$-wide channel, will meet perfectly out of phase. The same would be true for any integer multiple of $\lambda_{y}$. Conversely, for half integer multiples, the extra half period that it takes for the waves to meet leads to constructive interference.

\section{c. Channel width controls interference and conversion}

Tidally averaged conversion provides a single-valued quantity for each simulation that reflects the size of the response and allows easy comparison between all channel widths for a given obstacle and frequency. Specifically, we use the tidally averaged conversion integrated over the 40-km-long energy control volume shown in Fig. 1a. (Alternatively, we could have used baroclinic or barotropic flux divergence to reach the same conclusions.) When expressed per unit width, this quantity can be compared with the narrow-channel limit in which a subinertial channel without sidewall drag is equivalent to a two-dimensional, nonrotating ridge.

Curves of conversion as a function of channel width $w$ for all three obstacle shapes (Fig. 9) confirm the controlling role of the nondimensional parameter $w / \lambda_{y}$. Here we further demonstrate that the observed curves can be explained in large part by forgoing the threedimensional details and instead treating the system as the interference of two one-dimensional sinusoids that symbolize isopycnal perturbations.

Consider the isopycnal perturbations just east of the the obstacle. Let $Y_{K}(\mathrm{SE})$ and $Y_{R}(\mathrm{NE})$ refer to the perturbations in the Kelvin wave and ridge-trapped wave at their respective generation points. Then,

$$
\begin{gathered}
Y_{K}(\mathrm{SE})=\sin (2 \pi \omega t), \\
Y_{R}(\mathrm{NE})=\sin (-2 \pi \omega t),
\end{gathered}
$$

where $\omega$ is still the tidal frequency (e.g., $2 \pi / 44640$ s or $2 \pi / 55800 \mathrm{~s}$ ), and the negative sign for $Y_{R}$ accounts for the two waves being generated with opposite phase (section $5 b$ ).

The change in phase of the ridge-trapped wave by the time it reaches SE is simply $2 \pi$ times the channel width relative to the along-channel wavelength. Hence, at SE

$$
Y_{R}(\mathrm{SE})=\sin \left(-2 \pi \omega t+2 \pi w / \lambda_{y}\right) .
$$

For a Gaussian obstacle, the wave decays significantly as it travels along the ridge thereby reducing the interference. Empirically, we observe that the wave decays exponentially with a length scale comparable to $\lambda_{y}$. Let $Y_{\mathrm{GR}}$ describe this decaying wave:

$$
Y_{\mathrm{GR}}(\mathrm{SE})=\sin \left(-2 \pi \omega t+2 \pi w / \lambda_{y}\right) \exp \left(-w / \lambda_{y}\right)
$$

With the ridge-trapped and Kelvin waves interfering as described in section 5a, the Kelvin wave beyond the generation site, $Y_{K}(x \gg 0)$, will be the sum of the two waves at SE:

$$
Y_{K}(x \gg 0)=Y_{K}(\mathrm{SE})+Y_{R}(\mathrm{SE}),
$$

with $Y_{R}$ replaced by $Y_{\mathrm{GR}}$ for a Gaussian ridge.

The wave energy flux at $x \gg 0$ is proportional to the square of the isopycnal perturbations. Therefore, the tidally averaged wave energy flux per unit width for a non-Gaussian ridge is proportional to

$$
\frac{1}{w T} \int_{0}^{T}\left[Y_{K}(\mathrm{SE})+Y_{R}(\mathrm{SE})\right]^{2} d t,
$$

and similarly with $Y_{\mathrm{GR}}$ replacing $Y_{R}$ for a Gaussian ridge. Hereafter we presume that conversion is proportional to this wave energy flux (e.g., compare conversion and baroclinic flux divergence in Fig. 5). Hence, the expressions for conversion per unit width are

$$
\begin{array}{rlr}
\frac{C}{w C_{2 \mathrm{D}}} & =1, & w<0.5 \lambda_{y}, \\
& =\frac{0.250 \lambda_{y}}{w T} \int_{0}^{T}\left[Y_{K}(\mathrm{SE})+Y_{R}(\mathrm{SE})\right]^{2} d t ; & \text { tophat, } \quad w>0.5 \lambda_{y}, \\
& =\frac{0.386 \lambda_{y}}{w T} \int_{0}^{T}\left[Y_{K}(\mathrm{SE})+Y_{\mathrm{GR}}(\mathrm{SE})\right]^{2} d t ; & \text { Gaussian, } w>0.5 \lambda_{y},
\end{array}
$$

where $C_{2 \mathrm{D}}$ is per unit width conversion in the twodimensional, nonrotating limit and the numerators in front of the integrand are chosen to ensure that the expressions are continuous at $0.5 \lambda_{y}$. For channels narrower than $0.5 \lambda_{y}$, interference is not easily defined as there are not two clearly distinct waves. Furthermore, 


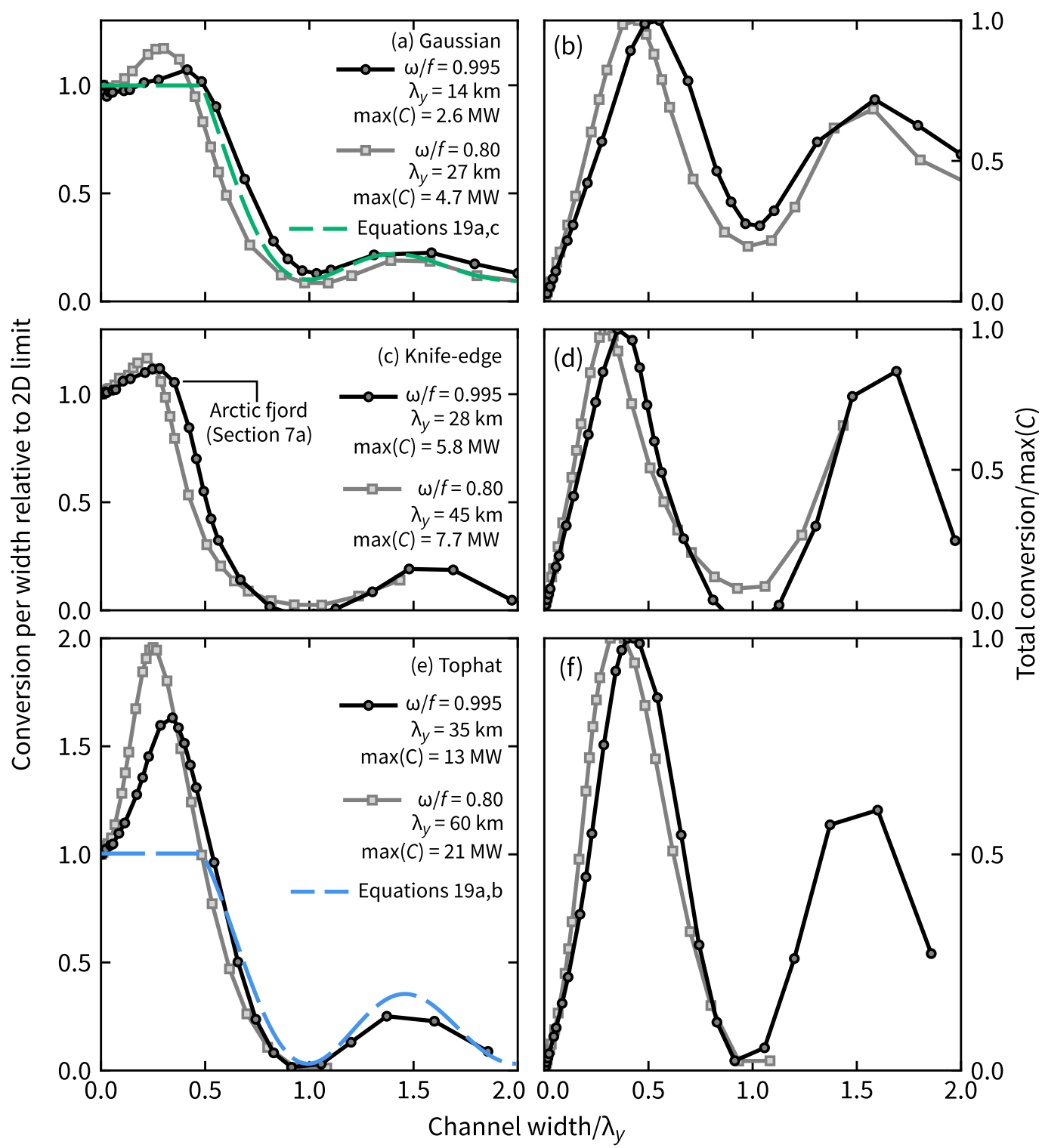

FIG. 9. Near-collapse of conversion curves when channel width is nondimensionalized by the along-obstacle wavelength. For a given obstacle and forcing frequency, $\max (C)$ refers to the maximum volume-integrated, tidally averaged conversion rate among all channel widths shown.

other factors that are described in section $5 \mathrm{~d}$ come into play. In this range, we simply equate conversion with the two-dimensional, nonrotating limit.

Considering their simplicity, these curves agree well with the values derived from the simulations (Fig. 9). Notably absent, however, is a prediction for the knifeedge obstacle. In this case, we cannot ignore interference above the crest between the waves on opposite sides of the obstacle and, consequently, a simple fit is no longer possible.

A limitation of our simple fits is they assume conversion is governed only by interference of the two trapped waves. Although this is typically true, counterexamples are channels wider than $10 L_{R}$ with a Gaussian obstacle (Fig. 5). In these cases, the along-ridge wave decays significantly before it reaches the other side of the channel. Consequently, tidal conversion due to dissipation in the evanescent response and lee waves near the ridge must be considered.

\section{d. Enhanced conversion in narrow channels}

In narrow channels $\left(\leq 0.5 \lambda_{y}\right)$, especially for the tophat obstacle, per unit width conversion may be larger than the two-dimensional, nonrotating limit. This is perhaps 


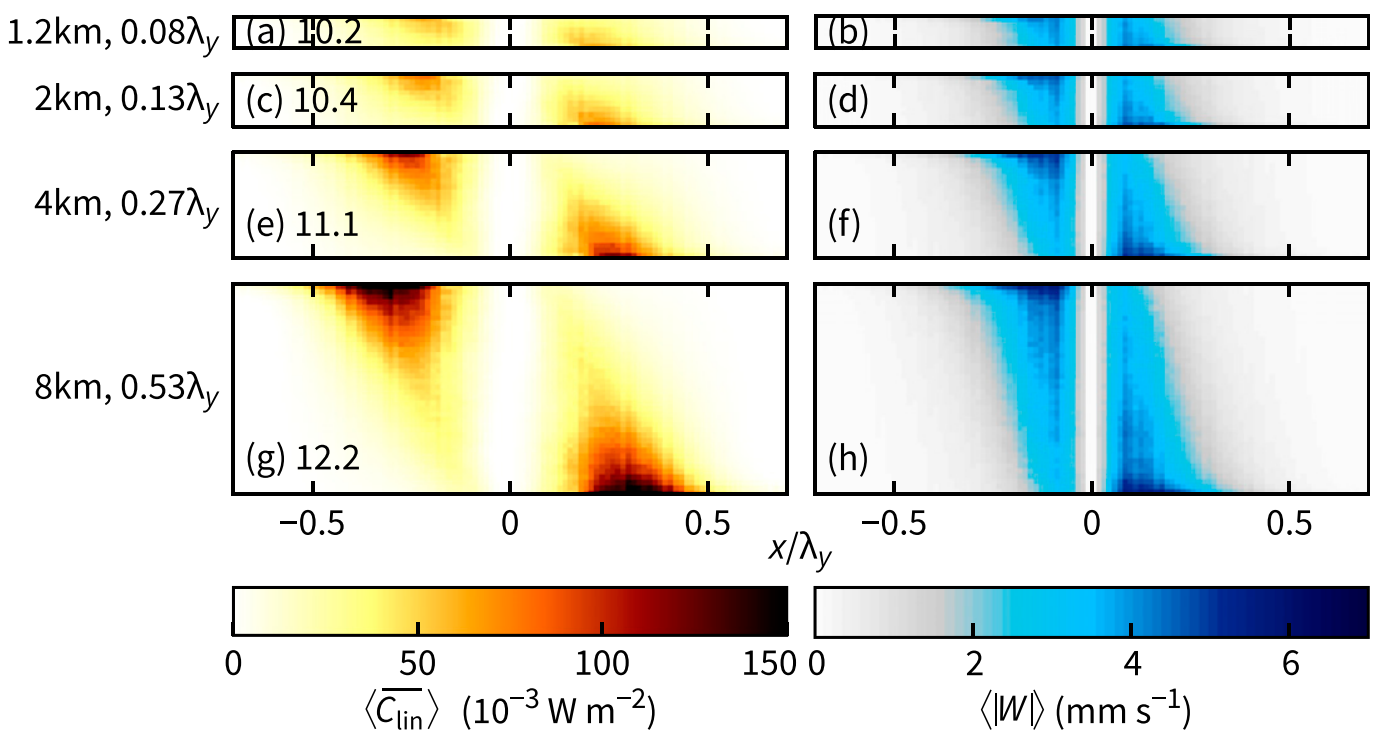

FIG. 10. Tidally averaged conversion and vertical barotropic speed in narrow channels. Within the range of channels shown, the spatial average of $\langle\bar{C}\rangle$ over the energy control volume (the values given beside the panel labels) increases with channel width. The vertical velocity $W$ [see Eq. (3)] is evaluated at middepth, and its absolute value is calculated before averaging. All simulations have a Gaussian obstacle and tidal frequency of $0.8 f$.

counterintuitive as in a two-dimensional, infinite-ridge scenario, conversion drops with increasing rotation as $\sqrt{1-f^{2} / \omega^{2}}$ and is zero if $\omega<f$ (section 1$)$. In a subinertial channel, the increase above the two-dimensional limit occurs, in part, because of how the barotropic tide propagates in the channel. As the barotropic tidal current impinges on an obstacle, the current above the obstacle is turned to its right as in Fig. 2 (see also chapter 3 of Pratt and Whitehead 2008). This induces barotropic convergence downstream of the obstacle at the righthand wall. Half of a tidal period later, the equivalent process occurs at the opposite end of the obstacle.

In all but the narrowest channels, the convergence of the barotropic flow at SE and NW owing to rotation is appreciable. As noted in section 2b, the vertical barotropic velocity $W$ is a measure of this convergence. Figure 10 demonstrates that the signal in $W$ (specifically its magnitude $|W|$ ) is concentrated near SE and NW. In moderately wide channels (e.g., $8 \mathrm{~km}$, Fig. $10 \mathrm{~h}$ ), $W$ can reach larger values than in narrow, quasi-twodimensional channels (e.g., $1.2 \mathrm{~km}$, Fig. 10b). Ultimately, it is this concentration of $W$, togther with a similar concentration of isopycnal perturbations, that allows tidal conversion to exceed the two-dimensional, nonrotating limit.

As we will show in the following sections, conversion is the dominant process by which energy is extracted from the barotropic tide. Furthermore, determining whether conversion is comparatively large or small is a prerequisite for an understanding of the full energy budget for a given channel. In a weakly responding channel, for example, near-obstacle turbulent dissipation will play a larger relative role.

\section{Barotropic and baroclinic energy budgets}

For superinertial latitudes in the weak forcing limit, loss of barotropic tidal energy over an obstacle is typically dominated by the generation of internal tides. As forcing is increased, other mechanisms come into play: high-frequency internal wave generation, bottom boundary layer dissipation, and near-ridge dissipation. In this section, we demonstrate that these same principles apply to subinertial internal tide generation in a channel. In particular, given the dominance of internal tides, a subinertial channel provides an obvious contrast to the subinertial infinite ridge in the weak-forcing limit in which barotropic energy loss is small as tidally averaged conversion occurs only via turbulent dissipation.

\section{a. Energetics with varying channel width}

To investigate how energy terms vary with channel width (Fig. 11), we use the Gaussian setup with the deepwater tidal current amplitudes fixed at $U_{0}=4 \mathrm{~cm} \mathrm{~s}^{-1}$ (see Table 1). The conversion curves, and consequently the flux divergence curves, are predominantly explained by the interference described in section 5 . Here we further consider curves for the dissipative terms $D_{0}, D^{\prime}$, and $\varepsilon$.

The barotropic component of bottom boundary layer dissipation $D_{0}$ shows the least variation with channel 

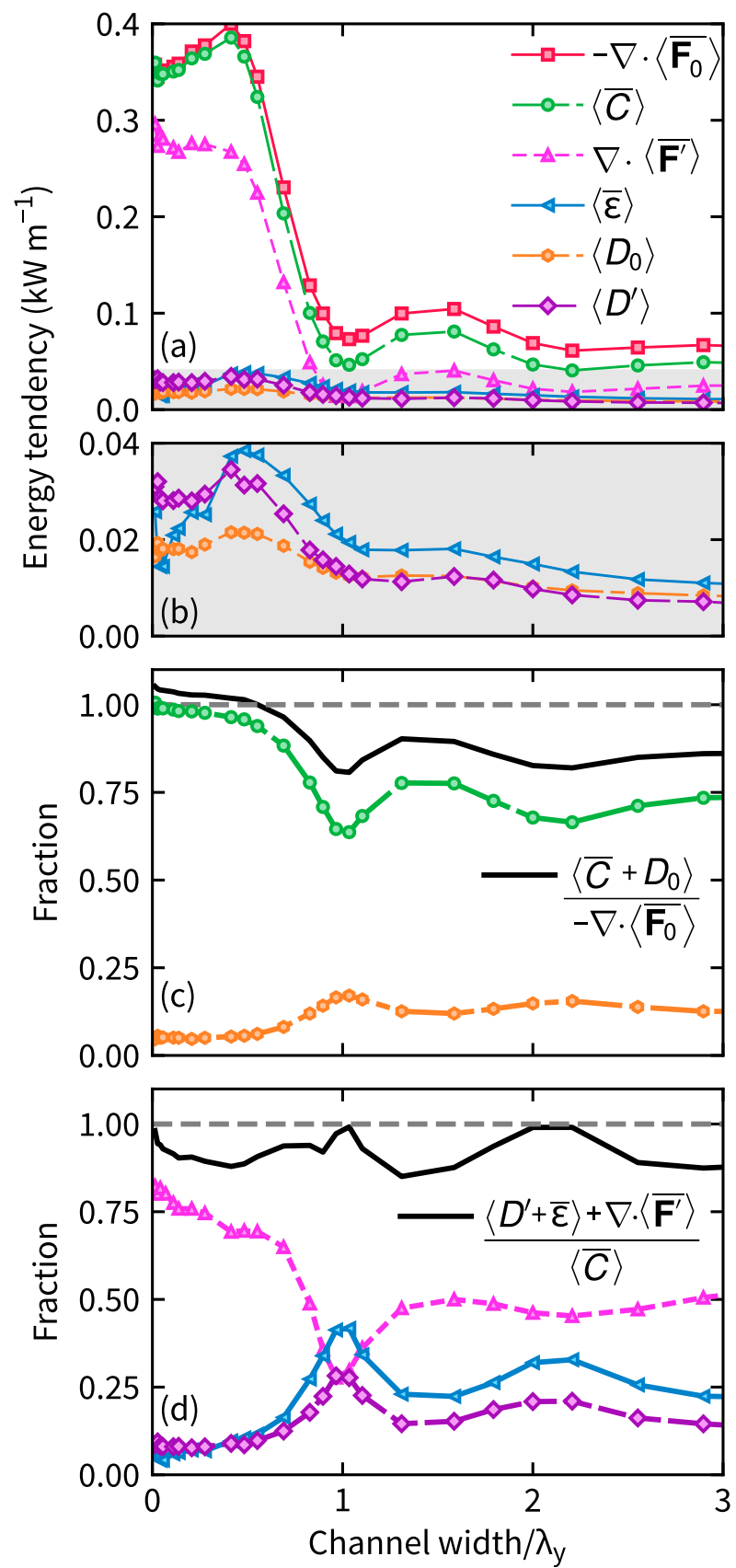

FIG. 11. The relevance of different energy terms for varying channel widths. Simulations all include a Gaussian obstacle and a tidal forcing of $U_{0}=4 \mathrm{~cm} \mathrm{~s}^{-1}$ at $\omega=0.995 f$. (a) Each component of the budget per unit width. (b) Zoomed variant of (a) to show the three small terms. The (c) barotropic and (d) baroclinic energy budgets. The black lines indicate the degree to which the terms shown close the individual budgets.

width of all six terms. Consequently, it plays a proportionately larger role in wider channels (Fig. 11c). Arguably, $D_{0}$ should show no variation with channel width given that it arises predominantly from barotropic flow normal to the obstacle. Some variation occurs, however, and is somewhat correlated with the total variance in the velocity fields.

The dissipative terms in the baroclinic energy budget, $D^{\prime}$ and $\varepsilon$, both display a trend that broadly corresponds to the conversion curve. For example, larger conversion equates to more variance in the baroclinic fields and consequently larger dissipation. The importance of these terms to the baroclinic energy budget (Fig. 11d) is largest when the channel width is an integer multiple of $\lambda_{y}$, but only because this is when the barotropic energy loss is at a minimum.

For many channel widths, up to $20 \%$ of both the barotropic and baroclinic energy budgets is not explicitly accounted for because of three factors. First, we assume the system is in a perfect tidally steady state. However, rates of change of barotropic and baroclinic energy densities over the tidal cycle account for as much as $3 \%-4 \%$ of each budget. Second, both $\nabla \cdot \mathbf{F}_{0}$ and $\bar{C}$ have large oscillations relative to their tidal averages, thereby amplifying any numerical issues related to resolution or discretization. In the wider channels, for example, peak-to-peak amplitudes of these quantities are order 50 times larger than their means. This effect is reduced in narrower channels, hence the better closure of the barotropic budget. Third, we include only explicit dissipation; numerical dissipation is not quantified.

\section{b. Energetics with varying tidal amplitude}

When tidal forcing is varied with channel width held fixed, the energetics are largely governed by power laws (Fig. 12). In this section, we fix channel width $(26 \mathrm{~km}$, $\left.1.8 \lambda_{y}\right)$ and forcing frequency $(\omega=0.995 f)$ and vary the deep-water tidal current amplitudes over the range $U_{0}=$ $1-24 \mathrm{~cm} \mathrm{~s}^{-1}$. For the barotropic energy budget, we empirically find that

$$
\begin{aligned}
\langle\bar{C}\rangle & \propto U_{0}^{2.07 \pm 0.02}, \\
\left\langle D_{0}\right\rangle & \propto U_{0}^{2.60 \pm 0.01} .
\end{aligned}
$$

The power law for $\langle\bar{C}\rangle$ is consistent with the twodimensional, superinertial prediction for knife-edge topography of $U_{0}^{2}$ (Llewellyn Smith and Young 2003). Despite the smaller exponent for $C$ compared to $D_{0}$, conversion is the dominant term in the barotropic energy budget across the forcing range we consider.

The exponent for the $D_{0}$ power law is not 3 as may be expected from the simple argument that it should arise from a drag force, $\propto U_{0}^{2}$, multiplied by the velocity (e.g., Taylor 1919). This argument does hold, however, when we account for the difference between the deep-water tidal current and the bottom velocity over the sill, where 

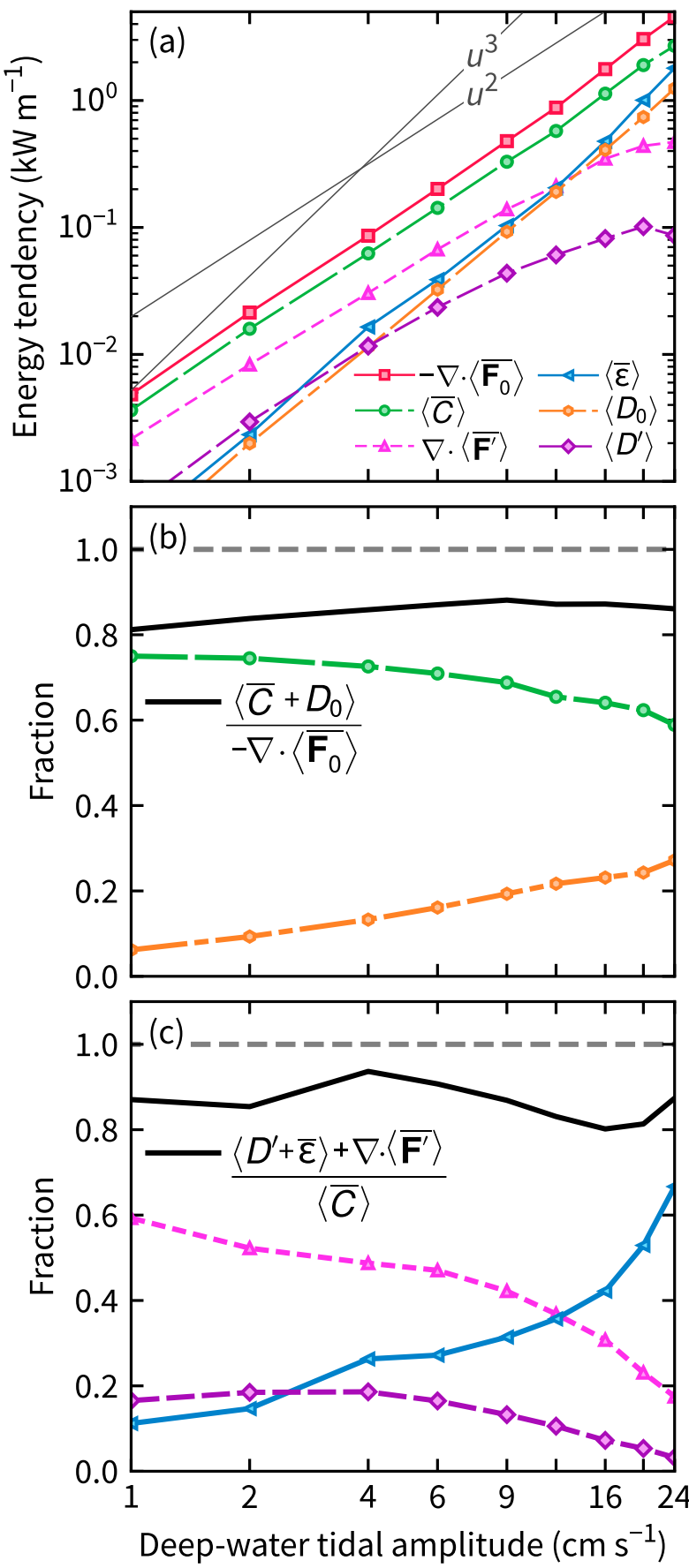

FIG. 12. The relevance of different energy terms for varying tidal forcing. Simulations all include a Gaussian obstacle in a channel of width $1.8 \lambda_{y}$. (a) Each component of the budget per unit width. Note the $\log -\log$ axis, which highlights the power-law scalings. The (b) barotropic and (c) baroclinic energy budgets. The black lines indicate the degree to which the terms shown close the individual budgets.
$D_{0}$ is concentrated. The effect of stratification is to inhibit near-bottom flow because vertical advection results in a transfer of kinetic to potential energy. Gemmrich and Klymak (2015), for example, find a power law relationship between the barotropic forcing velocity and the bottom boundary layer velocity of $U_{0} \propto u_{\mathrm{bbl}}^{0.87}$. (Their paper uses $y$, not $x$, as the cross-topography coordinate; we have adjusted their notation accordingly.) Applying their value in our case produces

$$
\left\langle D_{0}\right\rangle \propto u_{\mathrm{bbl}}^{3} \propto\left(U_{0}^{0.87}\right)^{3}=U_{0}^{2.6} .
$$

The baroclinic energy budget under varying forcing, rather than displaying a power-law nature, is better explained in terms of saturation: the baroclinic flux divergence and the baroclinic bottom boundary layer dissipation both taper off with increasing tidal forcing. Stronger forcing will produce larger baroclinic motions, but these cannot increase indefinitely. In the strongest forcing case $\left(U_{0}=24 \mathrm{~cm} \mathrm{~s}^{-1}\right)$ isopycnal displacements near the wall can reach $150 \mathrm{~m}$. The internal Kelvin waves that leave the energy control volume, however, are associated with displacements closer to $50 \mathrm{~m}$. For this simulation, much of the potential energy generated is lost either over the ridge or within the first wavelength of the Kelvin wave.

Energy loss over the ridge to turbulent dissipation is the most difficult parameter to explain. Vertical alongchannel slices (not shown) demonstrate that it predominantly occurs in breaking lee waves. Empirically, it obeys the following power law:

$$
\langle\bar{\varepsilon}\rangle \propto U_{0}^{2.59 \pm 0.05} .
$$

The similarity to the $D_{0}$ power law is likely not a coincidence. For superinertial tides over a sufficiently steep ridge, Klymak et al. (2010) would predict an exponent of 3. As we will describe, we expect a similar result for subinertial tides. As for $D_{0}$, the difference between the expected exponent of 3 and the actual exponent of 2.6 may result from a power law scaling of the barotropic velocity and the relevant velocity scale, which in this case is the velocity at the crest.

Klymak et al.'s (2010) parameterization assumes local dissipation of all energy contained in internal modes that travel slower than the tidal advection speed above the crest. The energy contained in these slower, higher modes is determined from a knife-edge model (Llewellyn Smith and Young 2003). This linear model, however, predicts zero energy for any mode when poleward of the critical latitude. Therefore we cannot quantitatively apply the parameterization. Nevertheless, their argument for a cubic power law remains valid. 
Briefly, the power law arises because the energy input into all modes slower than the critical mode $n_{c}$ scales as $U_{0}^{2} / n_{c}$ and $n_{c}$ is inversely proportional to $U_{0}$. As shown by Musgrave et al. [2016, see their Eqs. (2.31) and (2.32)], the energy fed into different modes behaves the same for subinertial and superinertial tides and is proportional to $U_{0}^{2}$ in both cases. There is not an obvious way to extend the concept of a critical mode to subinertial modes and thereby introduce an extra factor of $U_{0}$. We therefore, instead, appeal to the simple argument that dissipation will be proportional to the rate of energy supply, which is the product of the energy density and velocity (e.g., Tennekes and Lumley 1972, p. 20).

\section{Discussion}

\section{a. Practical application of the idealized system}

For superinertial latitudes, there are many methods for estimating the fate of energy lost from barotropic tides. Consider $q$, the fraction of energy dissipated locally relative to the energy converted from barotropic to baroclinic motion. The methods range from simple rules of thumb, such as the suggestion that $q$ is 0.3 (St. Laurent et al. 2002), to sophisticated recipes dependent on factors such as forcing, spectral characteristics of topography, and wave-wave interactions (Polzin 2009; Klymak et al. 2010). In this section, we reexamine our simulations to devise some general rules governing subinertial tide generation in channels that can be applied in practice.

The first step is to establish the along-ridge wavelength. In this study we provide a method and code for an arbitrarily shaped ridge with constant or vertically variable stratification. Presuming that the wavelength has been found, we now appeal to the two-dimensional, superinertial literature. In section $6 \mathrm{~b}$, we empirically found that the conversion scales with $U_{0}^{2}$, just as linear, two-dimensional theory suggests. This potentially implies that a first-order estimate of tidal conversion in a subinertial channel can be made using the analytical theory (or a two-dimensional simulation) with $f=0$, and then multiplying the appropriate fraction depending on the channel width relative to $\lambda_{y}$ (see Fig. 9).

A typical Arctic fjord, as noted by Cottier et al. (2010), is $10 \mathrm{~km}$ wide. Assume such a fjord features a steep sill comparable to our "knife-edge" ridge and has the same constant stratification we used in the simulations. Hence, we turn to Fig. 9c. If $\omega / f=0.995$, then $\lambda_{y}=28 \mathrm{~km}$ and the fjord width is $0.35 \lambda_{y}$. Reading off the curve indicates that conversion is 1.1 times the two-dimensional limit.
For some channels widths, especially $\sim 0.5 \lambda_{y}$, the conversion relative to the two-dimensional limit will depend sensitively on the exact obstacle and accuracy to which $\lambda_{y}$ is known. In such cases, a more practical goal may be to calculate upper and lower bounds for the expected conversion. Further, we note that we have not proven that a simple fractional scaling of the two-dimensional limit is always applicable. Confirming such a result would require many more simulations with different combinations of obstacles, tidal frequencies, and tidal forcings.

We now turn to diagnosing or predicting local dissipation fraction $q$, an often-sought quantity closely related to diapycnal diffusivity. In our argument to follow, we will ignore bottom boundary layer dissipation terms and thereby equate $q$ with $\varepsilon / C$. For subinertial latitudes in general, $q$ is close to 1 unless a boundary is present to act as a waveguide.

Despite covering only a small part of the total parameter space (channel width, tidal forcing, obstacle shape, among others), our simulations produced values of $q$ encompassing much of the 0 to 1 range. Nevertheless, $q$ follows three general rules. First, it is smallest in the narrowest channels. Second, it peaks when channel widths are integer multiples of $\lambda_{y}$. Third, it approaches 1 as forcing is increased to a level where the internal tides generated have isopycnal displacements comparable to the water depth. Such large displacements could also occur in two dimensions, but they are particularly prevalent here because rotation effectively shifts much of the potential energy toward the channel wall after its generation.

\section{b. The importance of three-dimensional processes}

The physics of tidal conversion poleward of the critical latitude changes significantly when a third dimension is introduced. In particular, convergence and divergence in the along-obstacle direction, a linear process, can generate nonzero tidally averaged conversion. In this study, channel walls induce the convergence and divergence, but any irregularities along the obstacle with a size comparable to the internal Rossby radius could do the same. Studies using only two-dimensional approaches may recognize this possibility, but nevertheless often appeal to a range of other explanations for nonzero conversion such as (i) the possibility that the critical latitude can shift poleward on the order of $100 \mathrm{~km}$ due to shear or nontraditional Coriolis effects (e.g., Lerczak et al. 2001; Gerkema and Shrira 2005); (ii) the empirical result that trapped energy decays with a scale of 3 days, thereby leading to an energy flux (Falahat and Nycander 2015); or (iii) that advection can generate high-frequency, freely propagating internal waves 
(e.g., Nakamura et al. 2000; Vlasenko et al. 2003; Rippeth et al. 2017).

Propagating, high-frequency waves are evident in our more strongly forced simulations. Energetically, however, they are a second-order effect relative to the Kelvin waves or near-ridge dissipation. As an example, we analyze a simulation where flow is supercritical over the ridge $\left(U_{0}=16 \mathrm{~cm} \mathrm{~s}^{-1}\right)$ in terms of low- and highfrequency components of $u^{\prime}$ and $p^{\prime}$ (Fig. 13). Specifically, we record $u^{\prime}$ and $p^{\prime}$ at $5 \mathrm{~min}$ intervals at a cross-channel slice $14 \mathrm{~km}$ from the crest of the obstacle and then lowand high-pass filter each using Butterworth filters with cutoff frequencies corresponding to half a tidal period $(6.2 \mathrm{~h})$. The low-frequency component of the signal $\left(\overline{u_{\text {low }}^{\prime} p_{\text {low }}^{\prime}}\right)$ captures $79 \%$ of the total, whereas the highfrequency component captures only $12 \%$ (cross terms account for the remainder). We recognize that this single example is far from proof that high-frequency waves are of minor importance here, especially when using a hydrostatic model with $200-\mathrm{m}$ resolution. However, this result is consistent with several others.

Using a two-dimensional, subinertial model, Musgrave et al. (2016) note that harmonics of a subinertial internal tide can propagate away but their energy content is small. Observationally, de Young and Pond (1989) found that high-frequency internal waves explain only $2 \%$ of the energy lost in a British Columbia fjord. (This example includes both superinertial $\mathrm{M}_{2}$ tides and subinertial $\mathrm{K}_{1}$ tides). Finally, when simulating internal waves around a midlatitude island, Masunaga et al. (2017) found that the subinertial diurnal internal tidal energy flux trapped around the island had an intensity roughly 100 times larger than that due to highfrequency waves.

When internal tides are trapped around an island, or similarly a plateau or seamount (e.g., Brink 1989), the majority of the energy will dissipate locally. In that respect, such three-dimensional systems are similar to a two-dimensional ridge. However, in the twodimensional case, dissipation within transient lee waves typically controls how much energy is extracted from the barotropic tide. Conversely, in the three-dimensional case, the amount of energy converted to a trapped-butpropagating internal tide is the controlling factor. We expect that more energy would be extracted for the three-dimensional case, especially when resonance occurs. For example, strong barotropic energy loss from the $\mathrm{K}_{1}$ tide in the Kuril Straits is linked to the generation of coastal-trapped waves (Tanaka et al. 2010). Similarly, Fer et al. (2015) describe the conversion at Yermak Plateau $\left(81^{\circ} \mathrm{N}\right)$ as substantial and demonstrate that the strongest conversion and dissipation occur at the steep sides of the plateau.

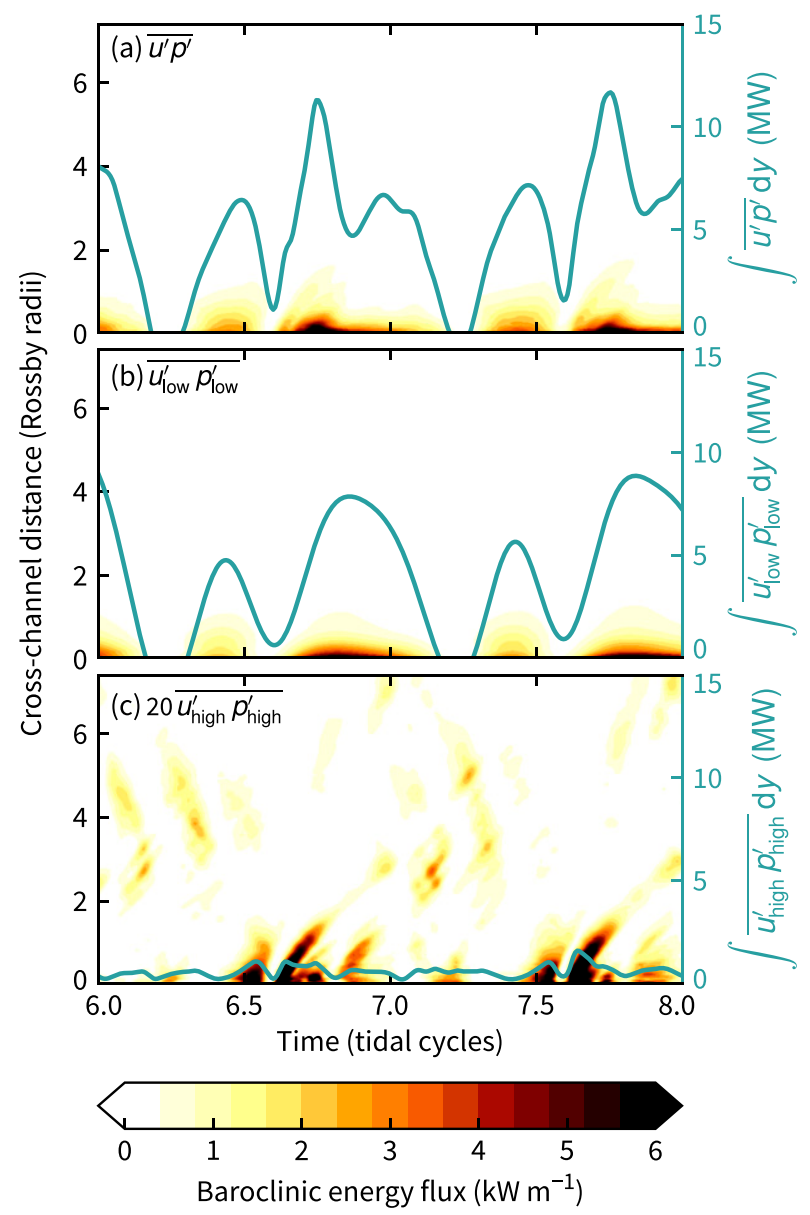

FIG. 13. (a)-(c) Contribution of low- and high-frequency internal waves to the total baroclinic flux over two tidal cycles. The values shown are taken from a cross-channel slice $14 \mathrm{~km}$ east of the crest of the Gaussian obstacle, and the deep-water tidal forcing was $16 \mathrm{~cm} \mathrm{~s}^{-1}$. Low- and high-frequency motions are separated at a frequency corresponding to a half tidal period. Note the factor of 20 in (c) without which the signal is imperceptible. The width integral (right-hand axis), however, is not adjusted.

Considering the behavior of waves trapped at steps and ridges (section 4), we note that the decay of internal tides is a plausible explanation for the observed correlation between bathymetric slope and turbulent dissipation in the Arctic (Rainville and Winsor 2008; Rippeth et al. 2015; Meyer et al. 2017), with the latter being a control on the magnitude of heat fluxes from warm Atlantic water at middepth. [See also Fer et al. (2016) for a similar correlation for the Antarctic.] This correlation may exist because steeper topography is a better waveguide for subinertial internal tides. Indeed, Musgrave et al. (2017) argue that this explains the large dissipation of diurnal internal tides at the Mendocino Ridge. Furthermore, this explanation, rather than a lee 
wave explanation, is consistent with the finding that turbulent mixing occurs even for relatively modest tidal currents (Rippeth et al. 2015).

\section{Conclusions}

We have used a suite of numerical simulations to demonstrate a simple mechanism that produces tidal conversion poleward of the critical latitude without any appeal to nonlinear behavior. Although we have focused on a channel, the simple requirement that the obstacle has either irregularities or a finite length means that subinertial tidal conversion can occur for other features such as plateaus and finite-length ridges. Idealized, three-dimensional modeling of such features in a tidal flow will likely prove fruitful and may elucidate the processes behind the strong subinertial conversion that has been identified in realistic simulations in locations such as Kuril Straits, Izu-Oshima Island, and Yermak Plateau. The role of the ratio of along-obstacle wave to obstacle size in other geometries without channel walls will be an interesting question.

The interference between the waves generated at each end of the obstacle in a channel modulates the energy extracted from the barotropic tide. The length scale over which this occurs, the wavelength of the along-ridge wave, is notably longer than the internal Rossby radius. At least with respect to energetics, channel width is better measured against this wavelength rather than the internal Rossby radius. Indeed, we reiterate the possibly counterintuitive finding that tidal conversion can be larger than suggested by an equivalent nonrotating, two-dimensional case even for channels up to approximately five internal Rossby radii wide.

Finally, we highlight a potentially misleading inference that can arise from studies that describe superinertial internal tides as freely propagating and subinertial tides as trapped. Yes, subinertial internal tides are trapped, but that should not imply that they cannot propagate. As long as they have a boundary to propagate beside, they are free to redistribute energy within the ocean, even if it is only a local redistribution.

Acknowledgments. This research was enabled in part by support provided by WestGrid (westgrid.ca) and Compute Canada (computecanada.ca). Ann Gargett provided a number of helpful suggestions on this paper, and Ruth Musgrave and an anonymous reviewer provided detailed comments that helped clarify several issues. Input for model simulations and output in a reduced form are available from kghughes.com/data.

\section{APPENDIX}

\section{Ridge-Trapped Kelvin Wave Derivation}

The step-trapped Kelvin wave described in section $4 \mathrm{~b}$ can be generalized from a single step to an arbitrary obstacle shape with or without a coastal boundary in a conceptually straightforward, albeit algebraically tedious, manner. Specifically, we assume the obstacle is composed of an arbitrary number of discontinuities $j=1, \ldots, J$ separating locally flat regions (Fig. A1). Matching conditions for the pressure $P$ and velocity $U$ perturbations are solved simultaneously at all discontinuities. Python code implementing this calculation is available at github.com/hugke729/ RidgeTrappedWave. Although it is not required in this paper, the code provided accepts vertically variable stratification.

The physical problem we seek to solve is

$$
P_{z z}+P_{\hat{x} \hat{x}}-\hat{l}^{2} P=0,
$$

where all dimensions $(x, y$, and $z)$ are scaled by the maximum depth such that $\min (z)=-1$. For a complete derivation see Chapman (1982, hereafter C82). Terms with a hat are further scaled by $R$ in the following way:

$$
(\hat{x}, \hat{y})=\left(\frac{x}{R}, \frac{y}{R}\right) ; \quad \hat{l}=l R ; \quad R^{2}=\frac{(N / f)^{2}-\omega^{2}}{1-\omega^{2}} .
$$

For consistency with $\mathrm{C} 82$, in this appendix $l$ denotes along-ridge wavenumber (termed $k_{y}$ elsewhere in this paper) and $\omega$ is nondimensional (termed $\omega^{*}$ elsewhere). The expression for $R^{2}$ is the full expression [see Eq. (4), not Eq. (B1), in C82].

Over each flat region, $P$ is a sum of modes whose coefficients $A_{n}, B_{n}, C_{n}^{j}$, and $D_{n}^{j}$ are to be found:

$$
\begin{aligned}
P_{0}= & \sum A_{n} \exp \left[\alpha_{n}\left(\hat{x}-\hat{x}_{1}\right)\right] \phi_{n}\left(h_{0}\right), \\
P_{J}= & \sum B_{n} \exp \left[-\beta_{n}\left(\hat{x}-\hat{x}_{J}\right)\right] \phi_{n}\left(h_{J}\right), \quad \text { and } \\
P_{j}= & \sum\left\{C_{n}^{j} \frac{\cosh \left[\gamma_{n}^{j}\left(\hat{x}-\hat{x}_{j+1 / 2}\right)\right]}{\cosh \left(\gamma_{n}^{j} \hat{L}_{j}\right)}\right. \\
& \left.+D_{n}^{j} \frac{\sinh \left[\gamma_{n}^{j}\left(\hat{x}-\hat{x}_{j+1 / 2}\right)\right]}{\sinh \left(\gamma_{n}^{j} \hat{L}_{j}\right)}\right\} \phi_{n}\left(h_{j}\right) .
\end{aligned}
$$

All summations are over $n$ from 0 to $n_{\max }=20$ (or any desired value). For the constant stratification case 


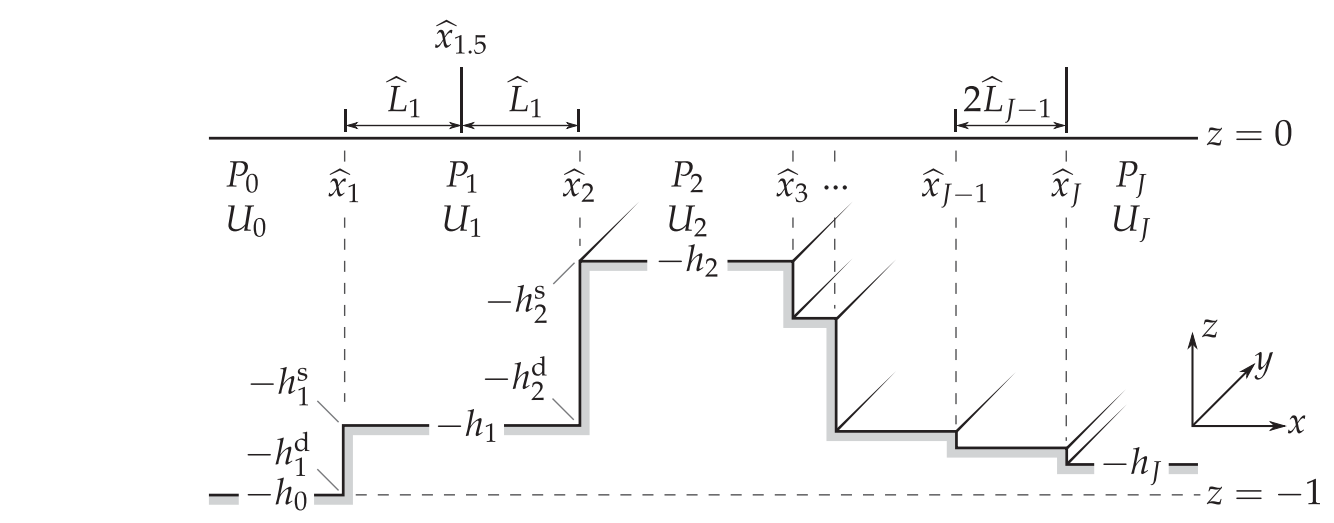

FIG. A1. Notation used in the derivation of the ridge-trapped wave traveling in the positive $y$ direction (into the page). In practice, the discontinuities would be chosen such that the ridge approximates a smooth surface, especially on the left-hand side. For clarity, this is not the case here.

$$
\begin{aligned}
\alpha_{n}^{2} & =\left(\frac{n \pi}{h_{0}}\right)^{2}+\hat{l}^{2}, \quad \beta_{n}^{2}=\left(\frac{n \pi}{h_{J}}\right)^{2}+\hat{l}^{2}, \\
\left(\gamma_{n}^{j}\right)^{2} & =\left(\frac{n \pi}{h_{j}}\right)^{2}+\hat{l}^{2},
\end{aligned}
$$

and $\phi_{n}(h)$ is the $n$th vertical mode shape for a depth $h$ :

$$
\phi_{n}(h)=\cos \left[\frac{n \pi}{h}(z+h)\right]
$$

The vertically variable stratification case is described near the end of this appendix.

The expressions in Eqs. (A3a) and (A3b) are reminiscent of the forms in C82, whereas Eq. (A3c) follows from Schmidt and Johnson (1997) who consider waves traveling beside a tophat ridge. This form is chosen such that both $P$ and its derivative simplify appreciably when evaluated at the discontinuities:

$$
\begin{aligned}
\left.P_{j}\right|_{\hat{x}=\hat{x}_{j+1}}= & \sum\left[C_{n}^{j}+D_{n}^{j}\right] \phi\left(h_{j}\right), \\
d P_{j} /\left.d \hat{x}\right|_{\hat{x}=\hat{x}_{j+1}}= & \sum\left[C_{n}^{j} \gamma_{n}^{j} \tanh \left(\gamma_{n}^{j} \hat{L}_{j}\right)\right. \\
& \left.+D_{n}^{j} \gamma_{n}^{j} \operatorname{coth}\left(\gamma_{n}^{j} \hat{L}_{j}\right)\right] \phi\left(h_{j}\right) .
\end{aligned}
$$

For a given discontinuity at $\hat{x}_{i}$, pressure is matched by equating $\left.P_{j}\right|_{\hat{x}_{j}}$ and $\left.P_{j}\right|_{\hat{x}_{j-1}}$, multiplying both sides by $\phi_{m}\left(h_{j}^{s}\right)$, and then integrating vertically from $-h_{j}^{s}$ to 0 . The subscript $s$ denotes the shallower of the two depths at $\hat{x}_{j}$ (see Fig. A1). Undertaking this at all discontinuities leads to the following set of equations:

$$
\begin{aligned}
& \int_{-h_{1}^{s}}^{0} \sum A_{n} \phi_{n}\left(h_{0}\right) \phi_{m}\left(h_{1}^{s}\right) d z \\
& \quad=\int_{-h_{1}^{s}}^{0} \sum\left[C_{n}^{1}-D_{n}^{1}\right] \phi_{n}\left(h_{1}\right) \phi_{m}\left(h_{1}^{s}\right) d z, \\
& \int_{-h_{j+1}^{s}}^{0} \sum\left[C_{n}^{j}+D_{n}^{j}\right] \phi_{n}\left(h_{j}\right) \phi_{m}\left(h_{j+1}^{s}\right) d z \\
& \quad=\int_{-h_{j+1}^{s}}^{0} \sum\left[C_{n}^{j+1}-D_{n}^{j+1}\right] \phi_{n}\left(h_{j+1}\right) \phi_{m}\left(h_{j+1}^{s}\right) d z, \quad \text { and } \\
& \int_{-h_{J}^{s}}^{0} \sum_{n}\left[C_{n}^{J-1}+D_{n}^{J-1}\right] \phi_{n}\left(h_{J-1}\right) \phi_{m}\left(h_{J}^{s}\right) d z \\
& =\int_{-h_{J}^{s}}^{0} \sum B_{n} \phi_{n}\left(h_{J}\right) \phi_{m}\left(h_{J}^{s}\right) d z .
\end{aligned}
$$

The equivalent process for $U=\omega P_{\hat{x}}-\hat{l} P$ is somewhat messier. Equate $U_{j}$ and $U_{j+1}$ at the discontinuity at $\hat{x}_{j+1}$, multiply by $\phi_{m}\left(h_{j+1}^{d}\right)$, and integrate vertically from $h_{j}$ and $h_{j+1}$ to zero. Integrating over different vertical ranges implicitly ensures that $U$ is zero right against the step (i.e., in the range $-h_{j+1}^{d}<z<-h_{j+1}^{d}$ at $\left.\hat{x}_{j+1}\right)$. The results are

$$
\int_{-h_{0}}^{0}\left[\omega \alpha_{n} A_{n}-\hat{l} A_{n}\right] \phi_{n}\left(h_{0}\right) \phi_{m}\left(h_{1}^{d}\right) d z=\int_{-h_{1}}^{0}\left[-\omega C_{n}^{1} \varepsilon_{n}^{1}+\omega D_{n}^{1} \zeta_{n}^{1}-\hat{l} C_{n}^{1}+\hat{l} D_{n}^{1}\right] \phi_{n}\left(h_{1}\right) \phi_{m}\left(h_{1}^{d}\right) d z
$$




$$
\begin{array}{r}
\int_{-h_{j}}^{0}\left[\omega C_{n}^{j} \varepsilon_{n}^{j}+\omega D_{n}^{j} \zeta_{n}^{j}-\hat{l} C_{n}^{j}-\hat{l} D_{n}^{j}\right] \phi_{n}\left(h_{j}\right) \phi_{m}\left(h_{j+1}^{d}\right) d z= \\
\int_{-h_{j+1}}^{0}\left[-\omega C_{n}^{j+1} \varepsilon_{n}^{j+1}+\omega D_{n}^{j+1} \zeta_{n}^{j}-\hat{l} C_{n}^{j+1}\right. \\
\\
\left.+\hat{l} D_{n}^{j+1}\right] \phi_{n}\left(h_{j+1}\right) \phi_{m}\left(h_{j+1}^{d}\right) d z, \quad \text { and } \\
\int_{-h_{J-1}}^{0}\left[\omega C_{n}^{J-1} \varepsilon_{n}^{J-1}+\omega D_{n}^{J-1} \zeta_{n}^{J-1}-\hat{l} C_{n}^{J-1}-\hat{l} D_{n}^{J-1}\right] \phi_{n}\left(h_{J-1}\right) \phi_{m}\left(h_{J}^{d}\right) d z=\int_{-h_{J}}^{0}\left[-\omega \beta_{n} B_{n}-\hat{l} B_{n}\right] \phi_{n}\left(h_{J}\right) \phi_{m}\left(h_{J}^{d}\right) d z,
\end{array}
$$

where

$$
\begin{aligned}
& \varepsilon_{n}^{j}=\gamma_{n}^{j} \tanh \left(\gamma_{n}^{j} \hat{L}_{j}\right), \\
& \zeta_{n}^{j}=\gamma_{n}^{j} \operatorname{coth}\left(\gamma_{n}^{j} \hat{L}_{j}\right) .
\end{aligned}
$$

To simplify notation, we introduce the following matrices

$$
E_{m n}^{j}=\int_{-h_{j+1}^{s}}^{0} \phi_{n}\left(h_{j}\right) \phi_{m}\left(h_{j+1}^{s}\right) d z
$$

$$
\begin{aligned}
& F_{m n}^{j}=\int_{-h_{j}^{s}}^{0} \phi_{n}\left(h_{j}\right) \phi_{m}\left(h_{j}^{s}\right) d z, \\
& G_{m n}^{j}=\int_{-h_{j}}^{0} \phi_{n}\left(h_{j}\right) \phi_{m}\left(h_{j+1}^{d}\right) d z, \quad \text { and } \\
& H_{m n}^{j}=\int_{-h_{j}}^{0} \phi_{n}\left(h_{j}\right) \phi_{m}\left(h_{j}^{d}\right) d z .
\end{aligned}
$$

Equations (A8)-(A13) can now be cast as a block matrix problem with terms involving $\omega$ on the right-hand side. An example with $J=3$ demonstrates the general form (with the $m n$ indices dropped for clarity):

$$
\begin{aligned}
& {\left[\begin{array}{cccccc}
E^{0} & -F^{1} & F^{1} & & & \\
& E^{1} & E^{1} & -F^{J-1} & F^{J-1} & \\
& & & E^{J-1} & E^{J-1} & -F^{J} \\
-\hat{l} G^{0} & \hat{l} H^{1} & -\hat{l} H^{1} & & & \\
& -\hat{l} G^{1} & -\hat{l} G^{1} & \hat{l} H^{J-1} & -\hat{l} H^{J-1} & \\
& & & -\hat{l} G^{J-1} & -\hat{l} G^{J-1} & \hat{l} H^{J}
\end{array}\right]\left[\begin{array}{c}
A \\
C^{1} \\
D^{1} \\
C^{J-1} \\
D^{J-1} \\
B
\end{array}\right]} \\
& =\omega\left[\begin{array}{cccccc} 
& & & & & \\
-G^{0} \alpha & -H^{1} \varepsilon^{1} & H^{1} \zeta^{1} & & & \\
& -G^{1} \varepsilon^{1} & -G^{1} \zeta^{1} & -H^{J-1} \varepsilon^{J-1} & H^{J-1} \zeta^{J-1} & \\
& & & -G^{J-1} \varepsilon^{J-1} & -G^{J-1} \zeta^{J-1} & -H^{J} \beta
\end{array}\right]\left[\begin{array}{c}
A \\
C^{1} \\
D^{1} \\
C^{J-1} \\
D^{J-1} \\
B
\end{array}\right] .
\end{aligned}
$$

For constant stratification, the matrices $\mathbf{E}, \mathbf{F}, \mathbf{G}$, and $\mathbf{H}$ have analytical forms based on the special matrices $\mathbf{P}$ and $\mathbf{Q}$ to be defined:

If $h_{j}$ is shallower than $h_{j+1}\left(h_{j+1}^{s}=h_{j}, h_{j+1}^{d}=h_{j+1}\right)$ :

$E_{m n}^{j}=\int_{-h_{j}}^{0} \phi_{n}\left(h_{j}\right) \phi_{m}\left(h_{j}\right) d z=P_{m n}\left(h_{j}\right)$,

$G_{m n}^{j}=\int_{-h_{j}}^{0} \phi_{n}\left(h_{j}\right) \phi_{m}\left(h_{j+1}\right) d z=Q_{m n}\left(h_{j}, h_{j+1}\right)$.

If $h_{j}$ is deeper than $h_{j+1}\left(h_{j+1}^{s}=h_{j+1}, h_{j+1}^{d}=h_{j}\right)$ :
$E_{m n}^{j}=\int_{-h_{j+1}}^{0} \phi_{n}\left(h_{j}\right) \phi_{m}\left(h_{j+1}\right) d z^{*}=Q_{n m}\left(h_{j+1}, h_{j}\right)$,

$G_{m n}^{j}=\int_{-h_{j}}^{0} \phi_{n}\left(h_{j}\right) \phi_{m}\left(h_{j}\right) d z=P_{m n}\left(h_{j}\right)$.

If $h_{j}$ is shallower than $h_{j-1}\left(h_{j}^{s}=h_{j}, h_{j}^{d}=h_{j-1}\right)$ :

$F_{m n}^{j}=\int_{-h_{j}}^{0} \phi_{n}\left(h_{j}\right) \phi_{m}\left(h_{j}\right) d z=P_{m n}\left(h_{j}\right)$, 
$H_{m n}^{j}=\int_{-h_{j}}^{0} \phi_{n}\left(h_{j}\right) \phi_{m}\left(h_{j-1}\right) d z=Q_{m n}\left(h_{j}, h_{j-1}\right)$.

If $h_{j}$ is deeper than $h_{j-1}\left(h_{j}^{s}=h_{j-1}, h_{j}^{d}=h_{j}\right)$ :

$$
\begin{aligned}
& F_{m n}^{j}=\int_{-h_{j-1}}^{0} \phi_{n}\left(h_{j}\right) \phi_{m}\left(h_{j-1}\right) d z^{*}=Q_{n m}\left(h_{j-1}, h_{j}\right), \\
& H_{m n}^{j}=\int_{-h_{j}}^{0} \phi_{n}\left(h_{j}\right) \phi_{m}\left(h_{j}\right) d z=P_{m n}\left(h_{j}\right) .
\end{aligned}
$$

Asterisks highlight transposed matrices $(\mathrm{nm}$, not $\mathrm{mn})$ as these are easy to miss. The special matrices $\mathbf{P}$ and $\mathbf{Q}$ are

$$
\begin{gathered}
P_{m n}(h)=\int_{-h}^{0} \cos \left[\frac{n \pi}{h}(z+h)\right] \cos \left[\frac{m \pi}{h}(z+h)\right] d z \\
=h \delta_{m n} / 2+h \delta_{00} / 2, \\
Q_{m n}\left(h_{1}, h_{2}\right)=\int_{-h_{1}}^{0} \cos \left[\frac{n \pi}{h_{1}}\left(z+h_{1}\right)\right] \cos \left[\frac{m \pi}{h_{2}}\left(z+h_{2}\right)\right] d z \\
= \begin{cases}h_{1}, & \text { if } m=n=0 \\
\frac{(-1)^{n}}{2} h_{1} \cos \left(\frac{h_{2} n \pi}{h_{1}}\right), & \text { if } m h_{1}=n h_{2} . \\
\frac{h_{1}^{2} h_{2} m \sin \left(m \pi \frac{h_{1}-h_{2}}{h_{2}}\right)}{h_{1}^{2} m^{2} \pi-h_{2}^{2} n^{2} \pi}, & \text { otherwise }\end{cases}
\end{gathered}
$$

As with the step-trapped Kelvin wave in section 4b, the block matrix equation [Eq. (A20)] is not a true eigenvalue equation because the eigenvalue $\omega$ is used in constructing the left-hand side. However, the problem can be solved in a small number of iterations by iteratively fitting a parabola to estimates of $(\omega, \hat{l})$ pairs.

To extend the problem to allow vertically variable stratification $N(z)$, we follow Durran (2000) who provides the starting point for the equivalent atmospheric problem. Equation (A1) becomes

$$
\left(\frac{P_{z}}{\tilde{N}^{2}}\right)_{z}+P_{\hat{x} \hat{x}}-\hat{l}^{2} P=0
$$

where $\tilde{N}=N / N_{0}$ with $N_{0}$ being the equivalent constant stratification that would produce the same internal Rossby radius. That is, $N_{0}=c \pi / H$ with the nonrotating wave speed $c$ found numerically (e.g., Kelly et al. 2013).

Vertical modes $\phi_{n}$ and eigenvalues $\gamma_{n}$ (or $\alpha_{n}$ or $\beta_{n}$ ) are calculated from the following Sturm-Liouville problem:

$$
\frac{d}{d z}\left(\frac{1}{\tilde{N}^{2}} \frac{d \phi}{d z}\right)+\left(\gamma^{2}-\hat{l}^{2}\right) \phi=0 .
$$

Boundary conditions of $d \phi / d z=0$ at the surface and seafloor are approximated with second-order, one-sided derivatives. Once the vertical eigenmodes and eigenvalues are found, the remainder of the calculation is the same as for the constant stratification case, albeit with the matching matrices evaluated numerically rather than using analytical forms.

To convert the ridge problem to a coastal problem where the seafloor goes to zero, we replace Eq. (A3b) with

$$
\begin{aligned}
P_{J}= & \sum B_{n}\left\{\exp \left[\beta_{n}\left(\hat{x}-\hat{x}_{J}-2 \hat{L}_{J}\right)\right]\right. \\
& \left.+\left(\frac{\omega \beta_{n}-\hat{l}}{\omega \beta_{n}+\hat{l}}\right) \exp \left[-\beta_{n}\left(\hat{x}-\hat{x}_{J}-2 \hat{L}_{J}\right)\right]\right\} \phi_{n}\left(h_{J}\right) .
\end{aligned}
$$

This form follows from Chapman and Hendershott (1982) and ensures that the velocity at the wall that intersects the seafloor is zero. Consequently, three of the submatrices in Eq. (A20) change:

$$
\begin{gathered}
-F^{J}, \hat{l} H^{J},-H^{J} \beta \rightarrow\left(-F^{J}, \hat{l} H^{J},+H^{J}\right)\left[\exp \left(-2 \beta \hat{L}_{J}\right)\right. \\
\left.+(1,1,-1) \times\left(\frac{\omega \beta-\hat{l}}{\omega \beta+\hat{l}}\right) \exp \left(2 \beta \hat{L}_{J}\right)\right] .
\end{gathered}
$$

Note that we have not strictly separated the $\omega$ and non$\omega$ terms onto the right and left sides, respectively.

\section{REFERENCES}

Adcroft, A., C. Hill, J.-M. Campin, J. Marshall, and P. Heimbach, 2004: Overview of the formulation and numerics of the MIT GCM. Proc. ECMWF Seminar Series on Numerical Methods: Recent Developments in Numerical Methods for Atmosphere and Ocean Modelling, Reading, United Kingdom, ECMWF, 139-149, https://www.ecmwf.int/node/7642.

Arneborg, L., P. Jansson, A. Staalstrøm, and G. Broström, 2017: Tidal energy loss, internal tide radiation, and local dissipation for two-layer tidal flow over a sill. J. Phys. Oceanogr., 47, 15211538, https://doi.org/10.1175/JPO-D-16-0148.1.

Balmforth, N. J., and T. Peacock, 2009: Tidal conversion by supercritical topography. J. Phys. Oceanogr., 39, 1965-1974, https://doi.org/10.1175/2009JPO4057.1.

Berntsen, J., J. Xing, and A. M. Davies, 2009: Numerical studies of flow over a sill: Sensitivity of the non-hydrostatic effects to the grid size. Ocean Dyn., 59, 1043-1059, https://doi.org/10.1007/ s10236-009-0227-0.

Brink, K. H., 1989: The effect of stratification on seamount-trapped waves. Deep-Sea Res., 36, 825-844, https://doi.org/10.1016/ 0198-0149(89)90031-9. 
_- 2006: Coastal-trapped waves with finite bottom friction. Dyn. Atmos. Oceans, 41, 172-190, https://doi.org/10.1016/ j.dynatmoce.2006.05.001.

Buijsman, M. C., S. Legg, and J. Klymak, 2012: Double-ridge internal tide interference and its effect on dissipation in Luzon Strait. J. Phys. Oceanogr., 42, 1337-1356, https://doi.org/10.1175/ JPO-D-11-0210.1.

Chapman, D. C., 1982: On the failure of Laplace's tidal equations to model subinertial motions at a discontinuity in depth. Dyn. Atmos. Oceans, 7, 1-16, https://doi.org/10.1016/0377-0265(82) 90002-1.

— geophysical ocean. Dyn. Atmos. Oceans, 7, 17-31, https:// doi.org/10.1016/0377-0265(82)90003-3.

Cottier, F. R., F. Nilsen, R. Skogseth, V. Tverberg, J. Skarðhamar, and H. Svendsen, 2010: Arctic fjords: A review of the oceanographic environment and dominant physical processes. Spec. Publ. Geol. Soc. London, 344, 35-50, https://doi.org/10.1144/ SP344.4.

Dale, A. C., and T. J. Sherwin, 1996: The extension of baroclinic coastal-trapped wave theory to superinertial frequencies. J. Phys. Oceanogr., 26, 2305-2315, https://doi.org/10.1175/ 1520-0485(1996)026<2305:TEOBCT >2.0.CO;2.

—, J. M. Huthnance, and T. J. Sherwin, 2001: Coastal-trapped waves and tides at near-inertial frequencies. J. Phys. Oceanogr., 31, 2958-2970, https://doi.org/10.1175/1520-0485(2001)031<2958: CTWATA $>2.0 . \mathrm{CO} ; 2$

de Young, B., and S. Pond, 1989: Partition of energy loss from the barotropic tide in fjords. J. Phys. Oceanogr., 19, 246-252, https:// doi.org/10.1175/1520-0485(1989)019<0246:POELFT>2.0.CO;2.

Durran, D. R., 2000: Small-amplitude coastally trapped disturbances and the reduced-gravity shallow-water approximation. Quart. J. Roy. Meteor. Soc., 126, 2671-2689, https://doi.org/ 10.1002/qj.49712656904.

Falahat, S., and J. Nycander, 2015: On the generation of bottomtrapped internal tides. J. Phys. Oceanogr., 45, 526-545, https:// doi.org/10.1175/JPO-D-14-0081.1.

Fer, I., M. Müller, and A. K. Peterson, 2015: Tidal forcing, energetics, and mixing near the Yermak Plateau. Ocean Sci., 11, 287-304, https://doi.org/10.5194/os-11-287-2015.

_ - E. Darelius, and K. B. Daae, 2016: Observations of energetic turbulence on the Weddell Sea continental slope. Geophys. Res. Lett., 43, 760-766, https://doi.org/10.1002/2015GL067349.

Freeland, H. J., and D. M. Farmer, 1980: Circulation and energetics of a deep, strongly stratified inlet. Can. J. Fish. Aquat. Sci., 37, 1398-1410, https://doi.org/10.1139/f80-179.

Gemmrich, J., and J. M. Klymak, 2015: Dissipation of internal wave energy generated on a critical slope. J. Phys. Oceanogr., 45, 2221-2238, https://doi.org/10.1175/JPO-D-14-0236.1.

Gerkema, T., and V. I. Shrira, 2005: Near-inertial waves on the "nontraditional" $\beta$ plane. J. Geophys. Res., 110, C01003, https://doi.org/10.1029/2004JC002519.

Hughes, K. G., J. M. Klymak, W. J. Williams, and H. Melling, 2018: Tidally modulated internal hydraulic flow and energetics in the central Canadian Arctic Archipelago. J. Geophys. Res., 123, 5210-5299, https://doi.org/10.1029/ 2018JC013770.

Huthnance, J. M., 1975: On trapped waves over a continental shelf. J. Fluid Mech., 69, 689-704, https://doi.org/10.1017/ S0022112075001632.

, 1978: On coastal trapped waves: Analysis and numerical calculation by inverse iteration. J. Phys. Oceanogr., 8, 74-92, https:// doi.org/10.1175/1520-0485(1978)008<0074:OCTWAA >2.0.CO;2.
_ L. Mysak, and D.-P. Wang, 1986: Coastal trapped waves. Baroclinic Processes on Continental Shelves, C. N. K. Mooers, Ed., Coastal and Estuarine Sciences, Vol. 3, Amer. Geophys. Union, 1-18.

Inall, M. E., and T. P. Rippeth, 2002: Dissipation of tidal energy and associated mixing in a wide fjord. Environ. Fluid Mech., 2, 219-240, https://doi.org/10.1023/A:1019846829875.

Inall, M., T. Rippeth, C. Griffiths, and P. Wiles, 2005: Evolution and distribution of TKE production and dissipation within stratified flow over topography. Geophys. Res. Lett., 32, L08607, https://doi.org/10.1029/2004GL022289.

Johnson, E. R., and J. T. Rodney, 2011: Spectral methods for coastal-trapped waves. Cont. Shelf Res., 31, 1481-1489, https:// doi.org/10.1016/j.csr.2011.06.009.

Kang, D., 2011: Energetics and dynamics of internal tides in Monterey Bay using numerical simulations. Ph.D. dissertation, Stanford University, $170 \mathrm{pp}$.

- and O. Fringer, 2012: Energetics of barotropic and baroclinic tides in the Monterey Bay area. J. Phys. Oceanogr., 42, 272290, https://doi.org/10.1175/JPO-D-11-039.1.

Kelly, S. M., and J. D. Nash, 2010: Internal-tide generation and destruction by shoaling internal tides. Geophys. Res. Lett., 37, L23611, https://doi.org/10.1029/2010GL045598.

_ , N. L. Jones, and J. D. Nash, 2013: A coupled model for Laplace's tidal equations in a fluid with one horizontal dimension and variable depth. J. Phys. Oceanogr., 43, 17801797, https://doi.org/10.1175/JPO-D-12-0147.1.

Klymak, J. M., and M. C. Gregg, 2004: Tidally generated turbulence over the Knight Inlet Sill. J. Phys. Oceanogr., 34, 1135-1151, https://doi.org/10.1175/1520-0485(2004)034<1135: TGTOTK $>2.0 . C O ; 2$.

— that resolve breaking internal waves. Ocean Modell., 33, 224234, https://doi.org/10.1016/j.ocemod.2010.02.005.

_- S. Legg, and R. Pinkel, 2010: A simple parameterization of turbulent tidal mixing near supercritical topography. J. Phys. Oceanogr., 40, 2059-2074, https://doi.org/10.1175/ 2010JPO4396.1.

__- M. Buijsman, S. Legg, and R. Pinkel, 2013: Parameterizing surface and internal tide scattering and breaking on supercritical topography: the one- and two-ridge cases. J. Phys. Oceanogr., 43, 1380-1397, https://doi.org/10.1175/ JPO-D-12-061.1.

— H. L. Simmons, D. Braznikov, S. Kelly, J. A. MacKinnon, M. H. Alford, R. Pinkel, and J. D. Nash, 2016: Reflection of linear internal tides from realistic topography: The Tasman continental slope. J. Phys. Oceanogr., 46, 3321-3337, https:// doi.org/10.1175/JPO-D-16-0061.1.

Lerczak, J. A., M. C. Hendershott, and C. D. Winant, 2001: Observations and modeling of coastal internal waves driven by a diurnal sea breeze. J. Geophys. Res., 106, 19715-19729, https://doi.org/10.1029/2001JC000811.

Llewellyn Smith, S. G., and W. R. Young, 2003: Tidal conversion at a very steep ridge. J. Fluid Mech., 495, 175-191, https:/ doi.org/10.1017/S0022112003006098.

Marshall, J., A. Adcroft, C. Hill, L. Perelman, and C. Heisey, 1997: A finite-volume, incompressible Navier Stokes model for studies of the ocean on parallel computers. J. Geophys. Res., 102, 5753-5766, https://doi.org/10.1029/96JC02775.

Masunaga, E., O. B. Fringer, Y. Kitade, H. Yamazaki, and S. M. Gallager, 2017: Dynamics and energetics of trapped diurnal internal Kelvin waves around a midlatitude island. J. Phys. Oceanogr., 47, 2479-2498, https://doi.org/10.1175/JPO-D-16-0167.1. 
McLaughlin, F. A., E. C. Carmack, R. G. Ingram, W. J. Williams, and C. Michel, 2004: Oceanography of the Northwest Passage. The Global Coastal Ocean: Interdisciplinary Regional Studies and Syntheses, A. R. Robinson and K. H. Brink, Eds., The Sea-Ideas and Observations on Progress in the Study of the Seas, Vol. 14B, Harvard University Press, 1211-1242.

Meyer, A., I. Fer, A. Sundfjord, and A. K. Peterson, 2017: Mixing rates and vertical heat fluxes north of Svalbard from Arctic winter to spring. J. Geophys. Res. Oceans, 122, 4569-4586, https://doi.org/10.1002/2016JC012441.

Mihanović, H., G. Beg Paklar, and M. Orlić, 2014: Resonant excitation of island-trapped waves in a shallow, seasonally stratified sea. Cont. Shelf Res., 77, 24-37, https://doi.org/ 10.1016/j.csr.2014.01.014.

Munk, W., and C. Wunsch, 1998: Abyssal recipes II: Energetics of tidal and wind mixing. Deep-Sea Res., 45, 1977-2010, https:// doi.org/10.1016/S0967-0637(98)00070-3.

Musgrave, R. C., R. Pinkel, J. A. MacKinnon, M. R. Mazloff, and W. R. Young, 2016: Stratified tidal flow over a tall ridge above and below the turning latitude. J. Fluid Mech., 793, 933-957, https://doi.org/10.1017/jfm.2016.150.

- J. A. MacKinnon, R. Pinkel, A. F. Waterhouse, J. Nash, and S. M. Kelly, 2017: The influence of subinertial internal tides on near-topographic turbulence at the Mendocino Ridge: Observations and modeling. J. Phys. Oceanogr., 47, 2139-2154, https://doi.org/10.1175/JPO-D-16-0278.1.

Nakamura, T., T. Awaji, T. Hatayama, K. Akitomo, T. Takizawa, T. Kono, Y. Kawasaki, and M. Fukasawa, 2000: The generation of large-amplitude unsteady lee waves by subinertial $K_{1}$ tidal flow: A possible vertical mixing mechanism in the Kuril Straits. J. Phys. Oceanogr., 30, 1601-1621, https://doi.org/ 10.1175/1520-0485(2000)030<1601:TGOLAU>2.0.CO;2.

Polzin, K. L., 2009: An abyssal recipe. Ocean Modell., 30, 298-309, https://doi.org/10.1016/j.ocemod.2009.07.006.

Pratt, L. J., and J. A. Whitehead, 2008: Rotating Hydraulics: Nonlinear Topographic Effects in the Ocean and Atmosphere. Springer, 589 pp., https://doi.org/10.1007/978-0-387-49572-9.

Rainville, L., and P. Winsor, 2008: Mixing across the Arctic Ocean: Microstructure observations during the Beringia 2005 Expedition. Geophys. Res. Lett., 35, L08606, https://doi.org/10.1029/ 2008 GL033532.

Rippeth, T. P., B. J. Lincoln, Y.-D. Lenn, J. A. M. Green, A. Sundfjord, and S. Bacon, 2015: Tide-mediated warming of Arctic halocline by Atlantic heat fluxes over rough topography. Nat. Geosci., 8, 191-194, https://doi.org/10.1038/ ngeo2350.

_ , V. Vlasenko, N. Stashchuk, B. D. Scannell, J. A. M. Green, B. J. Lincoln, and S. Bacon, 2017: Tidal conversion and mixing poleward of the critical latitude (an Arctic case study). Geophys. Res. Lett., 44, 12 349-12 357, https://doi.org/ 10.1002/2017GL075310.

Samelson, R. M., 1999: The vertical structure of linear coastaltrapped disturbances. Mon. Wea. Rev., 127, 201-213, https:// doi.org/10.1175/1520-0493(1999)127<0201:TVSOLC> 2.0. $\mathrm{CO} ; 2$

Sarkar, S., and A. Scotti, 2017: From topographic internal gravity waves to turbulence. Annu. Rev. Fluid Mech., 49, 195-220, https://doi.org/10.1146/annurev-fluid-010816-060013.

Schmidt, G. A., and E. Johnson, 1997: Instability in stratified rotating shear flow along ridges. J. Mar. Res., 55, 915-933, https:// doi.org/10.1357/0022240973224201.

St. Laurent, L. C., H. L. Simmons, and S. R. Jayne, 2002: Estimating tidally driven mixing in the deep ocean. Geophys. Res. Lett., 29, 2106, https://doi.org/10.1029/2002GL015633.

—- S. Stringer, C. Garrett, and D. Perrault-Joncas, 2003: The generation of internal tides at abrupt topography. Deep-Sea Res. I, 50, 987-1003, https://doi.org/10.1016/S0967-0637(03) 00096-7.

Stigebrandt, A., and J. Aure, 1989: Vertical mixing in basin waters of fjords. J. Phys. Oceanogr., 19, 917-926, https://doi.org/ 10.1175/1520-0485(1989)019<0917:VMIBWO>2.0.CO;2.

Støylen, E., and I. Fer, 2014: Tidally induced internal motion in an Arctic fjord. Nonlinear Processes Geophys., 21, 87-100, https:// doi.org/10.5194/npg-21-87-2014.

Tanaka, Y., T. Hibiya, Y. Niwa, and N. Iwamae, 2010: Numerical study of $\mathrm{K}_{1}$ internal tides in the Kuril straits. J. Geophys. Res., 115, C09016, https://doi.org/10.1029/2009JC005903.

Taylor, G. I., 1919: Tidal friction in the Irish Sea. Philos. Trans. Roy. Soc. London, A220, 1-33, https://doi.org/10.1098/ rsta.1920.0001.

Tennekes, H., and J. L. Lumley, 1972: A First Course in Turbulence. MIT Press, 300 pp.

Vlasenko, V., N. Stashchuk, K. Hutter, and K. Sabinin, 2003: Nonlinear internal waves forced by tides near the critical latitude. Deep-Sea Res. I, 50, 317-338, https://doi.org/10.1016/ S0967-0637(03)00018-9. 